\title{
An Introduction to MCMC for Machine Learning
}

\author{
CHRISTOPHE ANDRIEU \\ C.Andrieu@bristol.ac.uk \\ Department of Mathematics, Statistics Group, University of Bristol, University Walk, Bristol BS8 1TW, UK \\ NANDO DE FREITAS \\ nando@cs.ubc.ca \\ Department of Computer Science, University of British Columbia, 2366 Main Mall, Vancouver, \\ BC V6T 1Z4, Canada \\ ARNAUD DOUCET \\ doucet@ee.mu.oz.au \\ Department of Electrical and Electronic Engineering, University of Melbourne, Parkville, Victoria 3052, Australia \\ MICHAEL I. JORDAN \\ jordan@cs.berkeley.edu \\ Departments of Computer Science and Statistics, University of California at Berkeley, 387 Soda Hall, Berkeley, \\ CA 94720-1776, USA
}

\begin{abstract}
This purpose of this introductory paper is threefold. First, it introduces the Monte Carlo method with emphasis on probabilistic machine learning. Second, it reviews the main building blocks of modern Markov chain Monte Carlo simulation, thereby providing and introduction to the remaining papers of this special issue. Lastly, it discusses new interesting research horizons.
\end{abstract}

Keywords: Markov chain Monte Carlo, MCMC, sampling, stochastic algorithms

\section{Introduction}

A recent survey places the Metropolis algorithm among the ten algorithms that have had the greatest influence on the development and practice of science and engineering in the 20th century (Beichl \& Sullivan, 2000). This algorithm is an instance of a large class of sampling algorithms, known as Markov chain Monte Carlo (MCMC). These algorithms have played a significant role in statistics, econometrics, physics and computing science over the last two decades. There are several high-dimensional problems, such as computing the volume of a convex body in $d$ dimensions, for which MCMC simulation is the only known general approach for providing a solution within a reasonable time (polynomial in $d$ ) (Dyer, Frieze, \& Kannan, 1991; Jerrum \& Sinclair, 1996).

While convalescing from an illness in 1946, Stan Ulam was playing solitaire. It, then, occurred to him to try to compute the chances that a particular solitaire laid out with 52 cards would come out successfully (Eckhard, 1987). After attempting exhaustive combinatorial calculations, he decided to go for the more practical approach of laying out several solitaires at random and then observing and counting the number of successful plays. This idea of selecting a statistical sample to approximate a hard combinatorial problem by a much simpler problem is at the heart of modern Monte Carlo simulation. 
Stan Ulam soon realised that computers could be used in this fashion to answer questions of neutron diffusion and mathematical physics. He contacted John Von Neumann, who understood the great potential of this idea. Over the next few years, Ulam and Von Neumann developed many Monte Carlo algorithms, including importance sampling and rejection sampling. Enrico Fermi in the 1930's also used Monte Carlo in the calculation of neutron diffusion, and later designed the FERMIAC, a Monte Carlo mechanical device that performed calculations (Anderson, 1986). In the 1940's Nick Metropolis, a young physicist, designed new controls for the state-of-the-art computer (ENIAC) with Klari Von Neumann, John's wife. He was fascinated with Monte Carlo methods and this new computing device. Soon he designed an improved computer, which he named the MANIAC in the hope that computer scientists would stop using acronyms. During the time he spent working on the computing machines, many mathematicians and physicists (Fermi, Von Neumann, Ulam, Teller, Richtmyer, Bethe, Feynman, \& Gamow) would go to him with their work problems. Eventually in 1949, he published the first public document on Monte Carlo simulation with Stan Ulam (Metropolis \& Ulam, 1949). This paper introduces, among other ideas, Monte Carlo particle methods, which form the basis of modern sequential Monte Carlo methods such as bootstrap filters, condensation, and survival of the fittest algorithms (Doucet, de Freitas, \& Gordon, 2001). Soon after, he proposed the Metropolis algorithm with the Tellers and the Rosenbluths (Metropolis et al., 1953).

Many papers on Monte Carlo simulation appeared in the physics literature after 1953. From an inference perspective, the most significant contribution was the generalisation of the Metropolis algorithm by Hastings in 1970. Hastings and his student Peskun showed that Metropolis and the more general Metropolis-Hastings algorithms are particular instances of a large family of algorithms, which also includes the Boltzmann algorithm (Hastings, 1970; Peskun, 1973). They studied the optimality of these algorithms and introduced the formulation of the Metropolis-Hastings algorithm that we adopt in this paper. In the 1980's, two important MCMC papers appeared in the fields of computer vision and artificial intelligence (Geman \& Geman, 1984; Pearl, 1987). Despite the existence of a few MCMC publications in the statistics literature at this time, it is generally accepted that it was only in 1990 that MCMC made the first significant impact in statistics (Gelfand \& Smith, 1990). In the neural networks literature, the publication of Neal (1996) was particularly influential.

In the introduction to this special issue, we focus on describing algorithms that we feel are the main building blocks in modern MCMC programs. We should emphasize that in order to obtain the best results out of this class of algorithms, it is important that we do not treat them as black boxes, but instead try to incorporate as much domain specific knowledge as possible into their design. MCMC algorithms typically require the design of proposal mechanisms to generate candidate hypotheses. Many existing machine learning algorithms can be adapted to become proposal mechanisms (de Freitas et al., 2001). This is often essential to obtain MCMC algorithms that converge quickly. In addition to this, we believe that the machine learning community can contribute significantly to the solution of many open problems in the MCMC field. For this purpose, we have outlined several "hot" research directions at the end of this paper. Finally, readers are encouraged to consult the excellent texts of Chen, Shao, and Ibrahim (2001), Gilks, Richardson, and Spiegelhalter (1996), Liu (2001), Meyn and Tweedie (1993), Robert and Casella (1999) and review papers by Besag 
et al. (1995), Brooks (1998), Diaconis and Saloff-Coste (1998), Jerrum and Sinclair (1996), Neal (1993), and Tierney (1994) for more information on MCMC.

The remainder of this paper is organised as follows. In Part 2, we outline the general problems and introduce simple Monte Carlo simulation, rejection sampling and importance sampling. Part 3 deals with the introduction of MCMC and the presentation of the most popular MCMC algorithms. In Part 4, we describe some important research frontiers. To make the paper more accessible, we make no notational distinction between distributions and densities until the section on reversible jump MCMC.

\section{MCMC motivation}

MCMC techniques are often applied to solve integration and optimisation problems in large dimensional spaces. These two types of problem play a fundamental role in machine learning, physics, statistics, econometrics and decision analysis. The following are just some examples.

1. Bayesian inference and learning. Given some unknown variables $x \in \mathcal{X}$ and data $y \in \mathcal{Y}$, the following typically intractable integration problems are central to Bayesian statistics

(a) Normalisation. To obtain the posterior $p(x \mid y)$ given the prior $p(x)$ and likelihood $p(y \mid x)$, the normalising factor in Bayes' theorem needs to be computed

$$
p(x \mid y)=\frac{p(y \mid x) p(x)}{\int_{\mathcal{X}} p\left(y \mid x^{\prime}\right) p\left(x^{\prime}\right) d x^{\prime}} .
$$

(b) Marginalisation. Given the joint posterior of $(x, z) \in \mathcal{X} \times \mathcal{Z}$, we may often be interested in the marginal posterior

$$
p(x \mid y)=\int_{\mathcal{Z}} p(x, z \mid y) d z .
$$

(c) Expectation. The objective of the analysis is often to obtain summary statistics of the form

$$
\mathbb{E}_{p(x \mid y)}(f(x))=\int_{\mathcal{X}} f(x) p(x \mid y) d x
$$

for some function of interest $f: \mathcal{X} \rightarrow \mathbb{R}^{n_{f}}$ integrable with respect to $p(x \mid y)$. Examples of appropriate functions include the conditional mean, in which case $f(x)=x$, or the conditional covariance of $x$ where $f(x)=x x^{\prime}-\mathbb{E}_{p(x \mid y)}(x) \mathbb{E}_{p(x \mid y)}^{\prime}(x)$.

2. Statistical mechanics. Here, one needs to compute the partition function $Z$ of a system with states $s$ and Hamiltonian $E(s)$

$$
Z=\sum_{s} \exp \left[-\frac{E(s)}{k T}\right],
$$

where $k$ is the Boltzmann's constant and $T$ denotes the temperature of the system. Summing over the large number of possible configurations is prohibitively expensive (Baxter, 1982). Note that the problems of computing the partition function and the normalising constant in statistical inference are analogous. 
3. Optimisation. The goal of optimisation is to extract the solution that minimises some objective function from a large set of feasible solutions. In fact, this set can be continuous and unbounded. In general, it is too computationally expensive to compare all the solutions to find out which one is optimal.

4. Penalised likelihood model selection. This task typically involves two steps. First, one finds the maximum likelihood (ML) estimates for each model separately. Then one uses a penalisation term (for example MDL, BIC or AIC) to select one of the models. The problem with this approach is that the initial set of models can be very large. Moreover, many of those models are of not interest and, therefore, computing resources are wasted.

Although we have emphasized integration and optimisation, MCMC also plays a fundamental role in the simulation of physical systems. This is of great relevance in nuclear physics and computer graphics (Chenney \& Forsyth, 2000; Kalos \& Whitlock, 1986; Veach \& Guibas, 1997).

\subsection{The Monte Carlo principle}

The idea of Monte Carlo simulation is to draw an i.i.d. set of samples $\left\{x^{(i)}\right\}_{i=1}^{N}$ from a target density $p(x)$ defined on a high-dimensional space $\mathcal{X}$ (e.g. the set of possible configurations of a system, the space on which the posterior is defined, or the combinatorial set of feasible solutions). These $N$ samples can be used to approximate the target density with the following empirical point-mass function

$$
p_{N}(x)=\frac{1}{N} \sum_{i=1}^{N} \delta_{x^{(i)}}(x),
$$

where $\delta_{x^{(i)}}(x)$ denotes the delta-Dirac mass located at $x^{(i)}$. Consequently, one can approximate the integrals (or very large sums) $I(f)$ with tractable sums $I_{N}(f)$ that converge as follows

$$
I_{N}(f)=\frac{1}{N} \sum_{i=1}^{N} f\left(x^{(i)}\right) \underset{N \rightarrow \infty}{\stackrel{a . s .}{\longrightarrow}} I(f)=\int_{\mathcal{X}} f(x) p(x) d x .
$$

That is, the estimate $I_{N}(f)$ is unbiased and by the strong law of large numbers, it will almost surely (a.s.) converge to $I(f)$. If the variance (in the univariate case for simplicity) of $f(x)$ satisfies $\sigma_{f}^{2} \triangleq \mathbb{E}_{p(x)}\left(f^{2}(x)\right)-I^{2}(f)<\infty$, then the variance of the estimator $I_{N}(f)$ is equal to $\operatorname{var}\left(I_{N}(f)\right)=\frac{\sigma_{f}^{2}}{N}$ and a central limit theorem yields convergence in distribution of the error

$$
\sqrt{N}\left(I_{N}(f)-I(f)\right) \underset{N \rightarrow \infty}{\Longrightarrow} \mathcal{N}\left(0, \sigma_{f}^{2}\right)
$$

where $\Longrightarrow$ denotes convergence in distribution (Robert \& Casella, 1999; Section 3.2). The advantage of Monte Carlo integration over deterministic integration arises from the fact that the former positions the integration grid (samples) in regions of high probability. 
The $N$ samples can also be used to obtain a maximum of the objective function $p(x)$ as follows

$$
\hat{x}=\underset{x^{(i)} ; i=1, \ldots, N}{\arg \max } p\left(x^{(i)}\right)
$$

However, we will show later that it is possible to construct simulated annealing algorithms that allow us to sample approximately from a distribution whose support is the set of global maxima.

When $p(x)$ has standard form, e.g. Gaussian, it is straightforward to sample from it using easily available routines. However, when this is not the case, we need to introduce more sophisticated techniques based on rejection sampling, importance sampling and MCMC.

\subsection{Rejection sampling}

We can sample from a distribution $p(x)$, which is known up to a proportionality constant, by sampling from another easy-to-sample proposal distribution $q(x)$ that satisfies $p(x) \leq$ $M q(x), M<\infty$, using the accept/reject procedure describe in figure 1 (see also figure 2). The accepted $x^{(i)}$ can be easily shown to be sampled with probability $p(x)$ (Robert \&

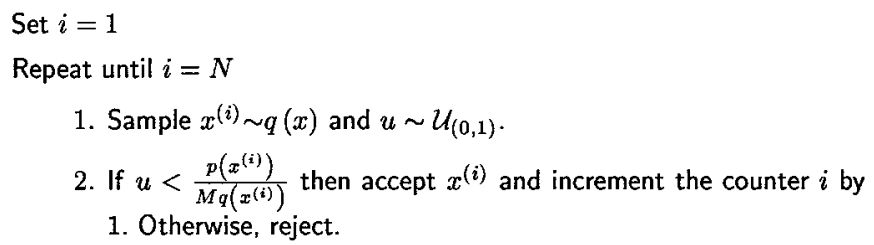

Figure 1. Rejection sampling algorithm. Here, $u \sim \mathcal{U}_{(0,1)}$ denotes the operation of sampling a uniform random variable on the interval $(0,1)$

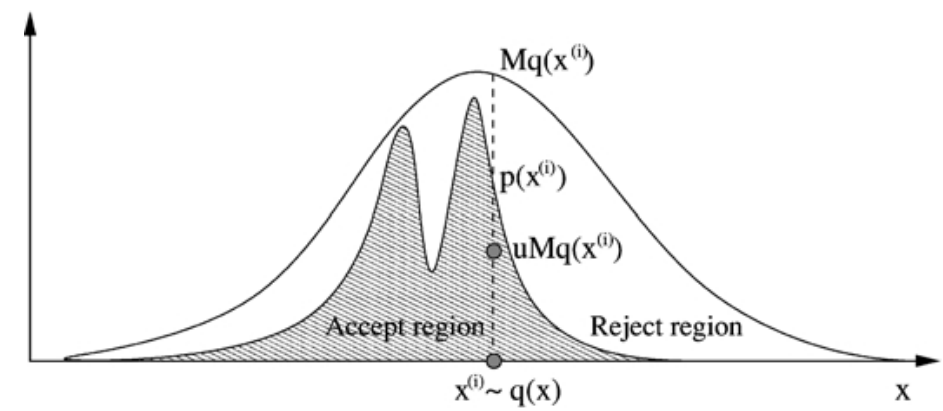

Figure 2. Rejection sampling: Sample a candidate $x^{(i)}$ and a uniform variable $u$. Accept the candidate sample if $u M q\left(x^{(i)}\right)<p\left(x^{(i)}\right)$, otherwise reject it. 
Casella, 1999, p. 49). This simple method suffers from severe limitations. It is not always possible to bound $p(x) / q(x)$ with a reasonable constant $M$ over the whole space $\mathcal{X}$. If $M$ is too large, the acceptance probability

$$
\operatorname{Pr}(x \text { accepted })=\operatorname{Pr}\left(u<\frac{p(x)}{M q(x)}\right)=\frac{1}{M}
$$

will be too small. This makes the method impractical in high-dimensional scenarios.

\subsection{Importance sampling}

Importance sampling is an alternative "classical" solution that goes back to the 1940's; see for example (Geweke, 1989; Rubinstein, 1981). Let us introduce, again, an arbitrary importance proposal distribution $q(x)$ such that its support includes the support of $p(x)$. Then we can rewrite $I(f)$ as follows

$$
I(f)=\int f(x) w(x) q(x) d x
$$

where $w(x) \triangleq \frac{p(x)}{q(x)}$ is known as the importance weight. Consequently, if one can simulate $N$ i.i.d. samples $\left\{x^{(i)}\right\}_{i=1}^{N}$ according to $q(x)$ and evaluate $w\left(x^{(i)}\right)$, a possible Monte Carlo estimate of $I(f)$ is

$$
\hat{I}_{N}(f)=\sum_{i=1}^{N} f\left(x^{(i)}\right) w\left(x^{(i)}\right)
$$

This estimator is unbiased and, under weak assumptions, the strong law of large numbers applies, that is $\hat{I}_{N}(f) \underset{N \rightarrow \infty}{\stackrel{a . s .}{\longrightarrow}} I(f)$. It is clear that this integration method can also be interpreted as a sampling method where the posterior density $p(x)$ is approximated by

$$
\hat{p}_{N}(x)=\sum_{i=1}^{N} w\left(x^{(i)}\right) \delta_{x^{(i)}}(x)
$$

and $\hat{I}_{N}(f)$ is nothing but the function $f(x)$ integrated with respect to the empirical measure $\hat{p}_{N}(x)$.

Some proposal distributions $q(x)$ will obviously be preferable to others. An important criterion for choosing an optimal proposal distribution is to find one that minimises the variance of the estimator $\hat{I}_{N}(f)$. The variance of $f(x) w(x)$ with respect to $q(x)$ is given by

$$
\operatorname{var}_{q(x)}(f(x) w(x))=\mathbb{E}_{q(x)}\left(f^{2}(x) w^{2}(x)\right)-I^{2}(f)
$$

The second term on the right hand side does not depend on $q(x)$ and hence we only need to minimise the first term, which according to Jensen's inequality has the following lower 
bound

$$
\mathbb{E}_{q(x)}\left(f^{2}(x) w^{2}(x)\right) \geq\left(\mathbb{E}_{q(x)}(|f(x)| w(x))\right)^{2}=\left(\int|f(x)| p(x) d x\right)^{2}
$$

This lower bound is attained when we adopt the following optimal importance distribution

$$
q^{\star}(x)=\frac{|f(x)| p(x)}{\int|f(x)| p(x) d x}
$$

The optimal proposal is not very useful in the sense that it is not easy to sample from $|f(x)| p(x)$. However, it tells us that high sampling efficiency is achieved when we focus on sampling from $p(x)$ in the important regions where $|f(x)| p(x)$ is relatively large; hence the name importance sampling.

This result implies that importance sampling estimates can be super-efficient. That is, for a given function $f(x)$, it is possible to find a distribution $q(x)$ that yields an estimate with a lower variance than when using a perfect Monte Carlo method, i.e. with $q(x)=p(x)$. This property is often exploited to evaluate the probability of rare events in communication networks (Smith, Shafi, \& Gao, 1997). There the quantity of interest is a tail probability (bit error rate) and hence $f(x)=\mathbb{I}_{E}(x)$ where $\mathbb{I}_{E}(x)=1$ if $x \in E$ and 0 otherwise (see figure 3 ). One could estimate the bit error rate more efficiently by sampling according to $q(x) \propto \mathbb{I}_{E}(x) p(x)$ than according to $q(x)=p(x)$. That is, it is wasteful to propose candidates in regions of no utility. In many applications, the aim is usually different in the sense that
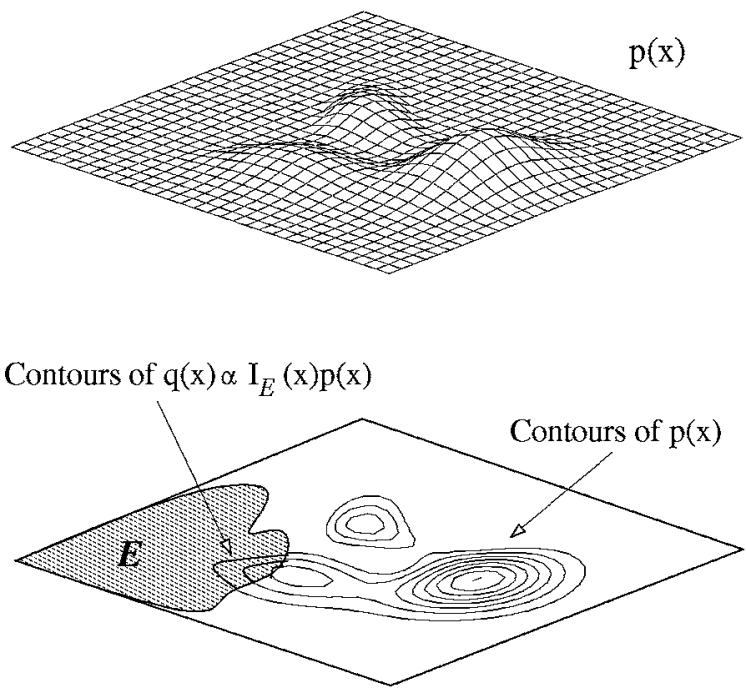

Figure 3. Importance sampling: one should place more importance on sampling from the state space regions that matter. In this particular example one is interested in computing a tail probability of error (detecting infrequent abnormalities). 
one wants to have a good approximation of $p(x)$ and not of a particular integral with respect to $p(x)$, so we often seek to have $q(x) \simeq p(x)$.

As the dimension of the $x$ increases, it becomes harder to obtain a suitable $q(x)$ from which to draw samples. A sensible strategy is to adopt a parameterised $q(x, \theta)$ and to adapt $\theta$ during the simulation. Adaptive importance sampling appears to have originated in the structural safety literature (Bucher, 1988), and has been extensively applied in the communications literature (Al-Qaq, Devetsikiotis, \& Townsend, 1995; Remondo et al., 2000). This technique has also been exploited recently in the machine learning community (de Freitas et al., 2000; Cheng \& Druzdzel, 2000; Ortiz \& Kaelbling, 2000; Schuurmans \& Southey, 2000). A popular adaptive strategy involves computing the derivative of the first term on the right hand side of Eq. (8)

$$
D(\theta)=\mathbb{E}_{q(x, \theta)}\left(f^{2}(x) w(x, \theta) \frac{\partial w(x, \theta)}{\partial \theta}\right)
$$

and then updating the parameters as follows

$$
\theta_{t+1}=\theta_{t}-\alpha \frac{1}{N} \sum_{i=1}^{N} f^{2}\left(x^{(i)}\right) w\left(x^{(i)}, \theta_{t}\right) \frac{\partial w\left(x^{(i)}, \theta_{t}\right)}{\partial \theta_{t}}
$$

where $\alpha$ is a learning rate and $x^{(i)} \sim q(x, \theta)$. Other optimisation approaches that make use of the Hessian are also possible.

When the normalising constant of $p(x)$ is unknown, it is still possible to apply the importance sampling method by rewriting $I(f)$ as follows:

$$
I(f)=\frac{\int f(x) w(x) q(x) d x}{\int w(x) q(x) d x}
$$

where $w(x) \propto \frac{p(x)}{q(x)}$ is now only known up to a normalising constant. The Monte Carlo estimate of $I(f)$ becomes

$$
\tilde{I}_{N}(f)=\frac{\frac{1}{N} \sum_{i=1}^{N} f\left(x^{(i)}\right) w\left(x^{(i)}\right)}{\frac{1}{N} \sum_{j=1}^{N} w\left(x^{(i)}\right)}=\sum_{i=1}^{N} f\left(x^{(i)}\right) \tilde{w}\left(x^{(i)}\right)
$$

where $\tilde{w}\left(x^{(i)}\right)$ is a normalised importance weight. For $N$ finite, $\tilde{I}_{N}(f)$ is biased (ratio of two estimates) but asymptotically, under weak assumptions, the strong law of large numbers applies, that is $\tilde{I}_{N}(f) \underset{N \rightarrow \infty}{\stackrel{\text { a.s. }}{\longrightarrow}} I(f)$. Under additional assumptions a central limit theorem can be obtained (Geweke, 1989). The estimator $\tilde{I}_{N}(f)$ has been shown to perform better than $\hat{I}_{N}(f)$ in some setups under squared error loss (Robert \& Casella, 1999).

If one is interested in obtaining $M$ i.i.d. samples from $\hat{p}_{N}(x)$, then an asymptotically $(N / M \rightarrow \infty)$ valid method consists of resampling $M$ times according to the discrete distribution $\hat{p}_{N}(x)$. This procedure results in $M$ samples $\tilde{x}^{(i)}$ with the possibility that $\tilde{x}^{(i)}=\tilde{x}^{(j)}$ 
for $i \neq j$. This method is known as sampling importance resampling (SIR) (Rubin, 1988). After resampling, the approximation of the target density is

$$
\tilde{p}_{M}(x)=\frac{1}{M} \sum_{i=1}^{M} \delta_{\tilde{x}^{(i)}}(x)
$$

The resampling scheme introduces some additional Monte Carlo variation. It is, therefore, not clear whether the SIR procedure can lead to practical gains in general. However, in the sequential Monte Carlo setting described in Section 4.3, it is essential to carry out this resampling step.

We conclude this section by stating that even with adaptation, it is often impossible to obtain proposal distributions that are easy to sample from and good approximations at the same time. For this reason, we need to introduce more sophisticated sampling algorithms based on Markov chains.

\section{MCMC algorithms}

MCMC is a strategy for generating samples $x^{(i)}$ while exploring the state space $\mathcal{X}$ using a Markov chain mechanism. This mechanism is constructed so that the chain spends more time in the most important regions. In particular, it is constructed so that the samples $x^{(i)}$ mimic samples drawn from the target distribution $p(x)$. (We reiterate that we use MCMC when we cannot draw samples from $p(x)$ directly, but can evaluate $p(x)$ up to a normalising constant.)

It is intuitive to introduce Markov chains on finite state spaces, where $x^{(i)}$ can only take $s$ discrete values $x^{(i)} \in \mathcal{X}=\left\{x_{1}, x_{2}, \ldots, x_{s}\right\}$. The stochastic process $x^{(i)}$ is called a Markov chain if

$$
p\left(x^{(i)} \mid x^{(i-1)}, \ldots, x^{(1)}\right)=T\left(x^{(i)} \mid x^{(i-1)}\right),
$$

The chain is homogeneous if $T \triangleq T\left(x^{(i)} \mid x^{(i-1)}\right)$ remains invariant for all $i$, with $\sum_{x^{(i)}} T\left(x^{(i)} \mid\right.$ $\left.x^{(i-1)}\right)=1$ for any $i$. That is, the evolution of the chain in a space $\mathcal{X}$ depends solely on the current state of the chain and a fixed transition matrix.

As an example, consider a Markov chain with three states $(s=3)$ and a transition graph as illustrated in figure 4 . The transition matrix for this example is

$$
T=\left[\begin{array}{ccc}
0 & 1 & 0 \\
0 & 0.1 & 0.9 \\
0.6 & 0.4 & 0
\end{array}\right]
$$

If the probability vector for the initial state is $\mu\left(x^{(1)}\right)=(0.5,0.2,0.3)$, it follows that $\mu\left(x^{(1)}\right) T=(0.2,0.6,0.2)$ and, after several iterations (multiplications by $\left.T\right)$, the product $\mu\left(x^{(1)}\right) T^{t}$ converges to $p(x)=(0.2,0.4,0.4)$. No matter what initial distribution $\mu\left(x^{(1)}\right)$ we use, the chain will stabilise at $p(x)=(0.2,0.4,0.4)$. This stability result plays a fundamental role in MCMC simulation. For any starting point, the chain will convergence to the 


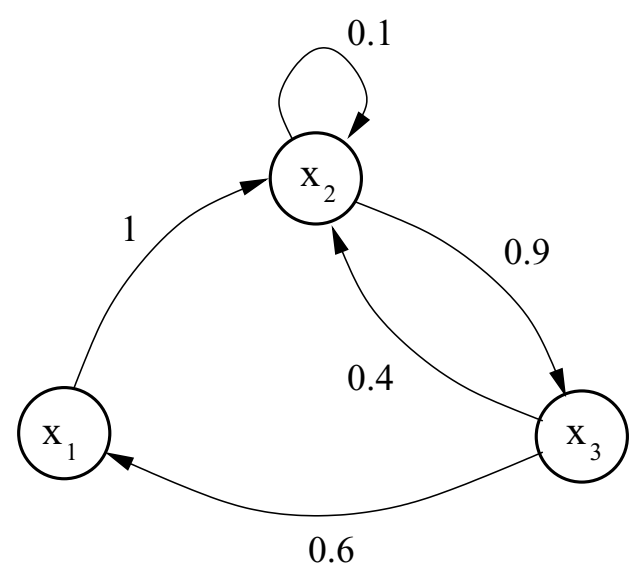

Figure 4. Transition graph for the Markov chain example with $\mathcal{X}=\left\{x_{1}, x_{2}, x_{3}\right\}$.

invariant distribution $p(x)$, as long as $T$ is a stochastic transition matrix that obeys the following properties:

1. Irreducibility. For any state of the Markov chain, there is a positive probability of visiting all other states. That is, the matrix $T$ cannot be reduced to separate smaller matrices, which is also the same as stating that the transition graph is connected.

2. Aperiodicity. The chain should not get trapped in cycles.

A sufficient, but not necessary, condition to ensure that a particular $p(x)$ is the desired invariant distribution is the following reversibility (detailed balance) condition

$$
p\left(x^{(i)}\right) T\left(x^{(i-1)} \mid x^{(i)}\right)=p\left(x^{(i-1)}\right) T\left(x^{(i)} \mid x^{(i-1)}\right) .
$$

Summing both sides over $x^{(i-1)}$, gives us

$$
p\left(x^{(i)}\right)=\sum_{x^{(i-1)}} p\left(x^{(i-1)}\right) T\left(x^{(i)} \mid x^{(i-1)}\right) .
$$

MCMC samplers are irreducible and aperiodic Markov chains that have the target distribution as the invariant distribution. One way to design these samplers is to ensure that detailed balance is satisfied. However, it is also important to design samplers that converge quickly. Indeed, most of our efforts will be devoted to increasing the convergence speed.

Spectral theory gives us useful insights into the problem. Notice that $p(x)$ is the left eigenvector of the matrix $T$ with corresponding eigenvalue 1. In fact, the Perron-Frobenius theorem from linear algebra tells us that the remaining eigenvalues have absolute value less than 1 . The second largest eigenvalue, therefore, determines the rate of convergence of the chain, and should be as small as possible.

The concepts of irreducibility, aperiodicity and invariance can be better appreciated once we realise the important role that they play in our lives. When we search for information on 
the World-Wide Web, we typically follow a set of links (Berners-Lee et al., 1994). We can interpret the webpages and links, respectively, as the nodes and directed connections in a Markov chain transition graph. Clearly, we (say, the random walkers on the Web) want to avoid getting trapped in cycles (aperiodicity) and want to be able to access all the existing webpages (irreducibility). Let us consider, now, the popular information retrieval algorithm used by the search engine Google, namely PageRank (Page et al., 1998). PageRank requires the definition of a transition matrix with two components $T=L+E$. $L$ is a large link matrix with rows and columns corresponding to web pages, such that the entry $L_{i, j}$ represents the normalised number of links from web page $i$ to web page $j$. $E$ is a uniform random matrix of small magnitude that is added to $L$ to ensure irreducibility and aperiodicity. That is, the addition of noise prevents us from getting trapped in loops, as it ensures that there is always some probability of jumping to anywhere on the Web. From our previous discussion, we have

$$
p\left(x^{(i+1)}\right)[L+E]=p\left(x^{i}\right)
$$

where, in this case, the invariant distribution (eigenvector) $p(x)$ represents the rank of a webpage $x$. Note that it is possible to design more interesting transition matrices in this setting. As long as one satisfies irreducibility and aperiodicity, one can incorporate terms into the transition matrix that favour particular webpages or that bias the search in useful ways.

In continuous state spaces, the transition matrix $T$ becomes an integral kernel $K$ and $p(x)$ becomes the corresponding eigenfunction

$$
\int p\left(x^{(i)}\right) K\left(x^{(i+1)} \mid x^{(i)}\right) d x^{(i)}=p\left(x^{(i+1)}\right)
$$

The kernel $K$ is the conditional density of $x^{(i+1)}$ given the value $x^{(i)}$. It is a mathematical representation of a Markov chain algorithm. In the following subsections we describe various of these algorithms.

\subsection{The Metropolis-Hastings algorithm}

The Metropolis-Hastings (MH) algorithm is the most popular MCMC method (Hastings, 1970; Metropolis et al., 1953). In later sections, we will see that most practical MCMC algorithms can be interpreted as special cases or extensions of this algorithm.

An MH step of invariant distribution $p(x)$ and proposal distribution $q\left(x^{\star} \mid x\right)$ involves sampling a candidate value $x^{\star}$ given the current value $x$ according to $q\left(x^{\star} \mid x\right)$. The Markov chain then moves towards $x^{\star}$ with acceptance probability $\mathcal{A}\left(x, x^{\star}\right)=\min \left\{1,\left[p(x) q\left(x^{\star} \mid\right.\right.\right.$ $\left.x)]^{-1} p\left(x^{\star}\right) q\left(x \mid x^{\star}\right)\right\}$, otherwise it remains at $x$. The pseudo-code is shown in figure 5 , while figure 6 shows the results of running the MH algorithm with a Gaussian proposal distribution $q\left(x^{\star} \mid x^{(i)}\right)=\mathcal{N}\left(x^{(i)}, 100\right)$ and a bimodal target distribution $p(x) \propto 0.3 \exp \left(-0.2 x^{2}\right)+$ $0.7 \exp \left(-0.2(x-10)^{2}\right)$ for 5000 iterations. As expected, the histogram of the samples approximates the target distribution. 


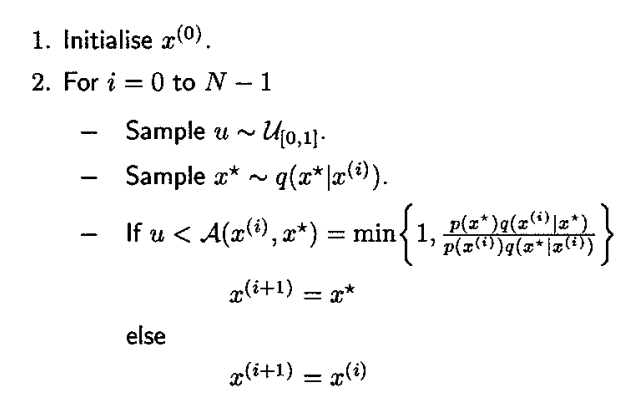

Figure 5. Metropolis-Hastings algorithm.
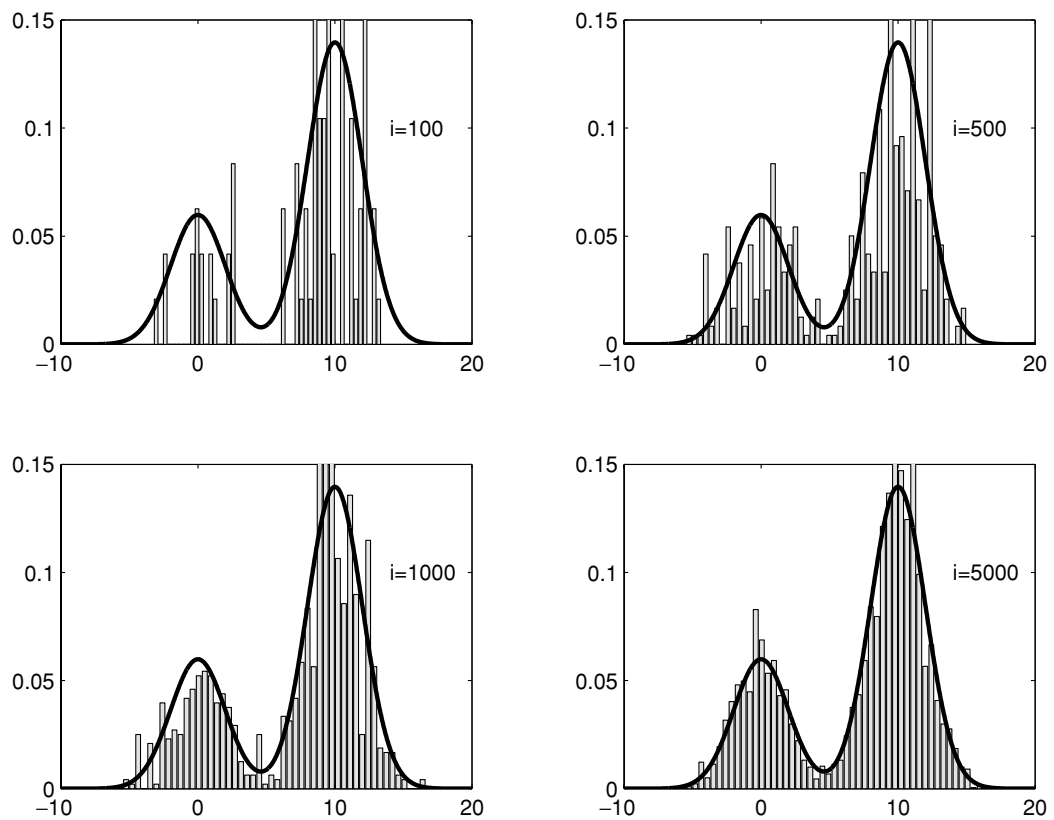

Figure 6. Target distribution and histogram of the MCMC samples at different iteration points.

The MH algorithm is very simple, but it requires careful design of the proposal distribution $q\left(x^{\star} \mid x\right)$. In subsequent sections, we will see that many MCMC algorithms arise by considering specific choices of this distribution. In general, it is possible to use suboptimal inference and learning algorithms to generate data-driven proposal distributions.

The transition kernel for the $\mathrm{MH}$ algorithm is

$$
K_{\mathrm{MH}}\left(x^{(i+1)} \mid x^{(i)}\right)=q\left(x^{(i+1)} \mid x^{(i)}\right) \mathcal{A}\left(x^{(i)}, x^{(i+1)}\right)+\delta_{x^{(i)}}\left(x^{(i+1)}\right) r\left(x^{(i)}\right),
$$


where $r\left(x^{(i)}\right)$ is the term associated with rejection

$$
r\left(x^{(i)}\right)=\int_{\mathcal{X}} q\left(x^{\star} \mid x^{(i)}\right)\left(1-\mathcal{A}\left(x^{(i)}, x^{\star}\right)\right) d x^{\star} .
$$

It is fairly easy to prove that the samples generated by $\mathrm{MH}$ algorithm will mimic samples drawn from the target distribution asymptotically. By construction, $K_{\mathrm{MH}}$ satisfies the detailed balance condition

$$
p\left(x^{(i)}\right) K_{\mathrm{MH}}\left(x^{(i+1)} \mid x^{(i)}\right)=p\left(x^{(i+1)}\right) K_{\mathrm{MH}}\left(x^{(i)} \mid x^{(i+1)}\right)
$$

and, consequently, the $\mathrm{MH}$ algorithm admits $p(x)$ as invariant distribution. To show that the $\mathrm{MH}$ algorithm converges, we need to ensure that there are no cycles (aperiodicity) and that every state that has positive probability can be reached in a finite number of steps (irreducibility). Since the algorithm always allows for rejection, it follows that it is aperiodic. To ensure irreducibility, we simply need to make sure that the support of $q(\cdot)$ includes the support of $p(\cdot)$. Under these conditions, we obtain asymptotic convergence (Tierney, 1994, Theorem 3, p. 1717). If the space $\mathcal{X}$ is small (for example, bounded in $\mathbb{R}^{n}$ ), then it is possible to use minorisation conditions to prove uniform (geometric) ergodicity (Meyn $\&$ Tweedie, 1993). It is also possible to prove geometric ergodicity using Foster-Lyapunov drift conditions (Meyn \& Tweedie, 1993; Roberts \& Tweedie, 1996).

The independent sampler and the Metropolis algorithm are two simple instances of the $\mathrm{MH}$ algorithm. In the independent sampler the proposal is independent of the current state, $q\left(x^{\star} \mid x^{(i)}\right)=q\left(x^{\star}\right)$. Hence, the acceptance probability is

$$
\mathcal{A}\left(x^{(i)}, x^{\star}\right)=\min \left\{1, \frac{p\left(x^{\star}\right) q\left(x^{(i)}\right)}{p\left(x^{(i)}\right) q\left(x^{\star}\right)}\right\}=\min \left\{1, \frac{w\left(x^{\star}\right)}{w\left(x^{(i)}\right)}\right\} .
$$

This algorithm is close to importance sampling, but now the samples are correlated since they result from comparing one sample to the other. The Metropolis algorithm assumes a symmetric random walk proposal $q\left(x^{\star} \mid x^{(i)}\right)=q\left(x^{(i)} \mid x^{\star}\right)$ and, hence, the acceptance ratio simplifies to

$$
\mathcal{A}\left(x^{(i)}, x^{\star}\right)=\min \left\{1, \frac{p\left(x^{\star}\right)}{p\left(x^{(i)}\right)}\right\} .
$$

Some properties of the $\mathrm{MH}$ algorithm are worth highlighting. Firstly, the normalising constant of the target distribution is not required. We only need to know the target distribution up to a constant of proportionality. Secondly, although the pseudo-code makes use of a single chain, it is easy to simulate several independent chains in parallel. Lastly, the success or failure of the algorithm often hinges on the choice of proposal distribution. This is illustrated in figure 7. Different choices of the proposal standard deviation $\sigma^{\star}$ lead to very different results. If the proposal is too narrow, only one mode of $p(x)$ might be visited. On the other hand, if it is too wide, the rejection rate can be very high, resulting in high correlations. If all the modes are visited while the acceptance probability is high, the chain is said to "mix" well. 


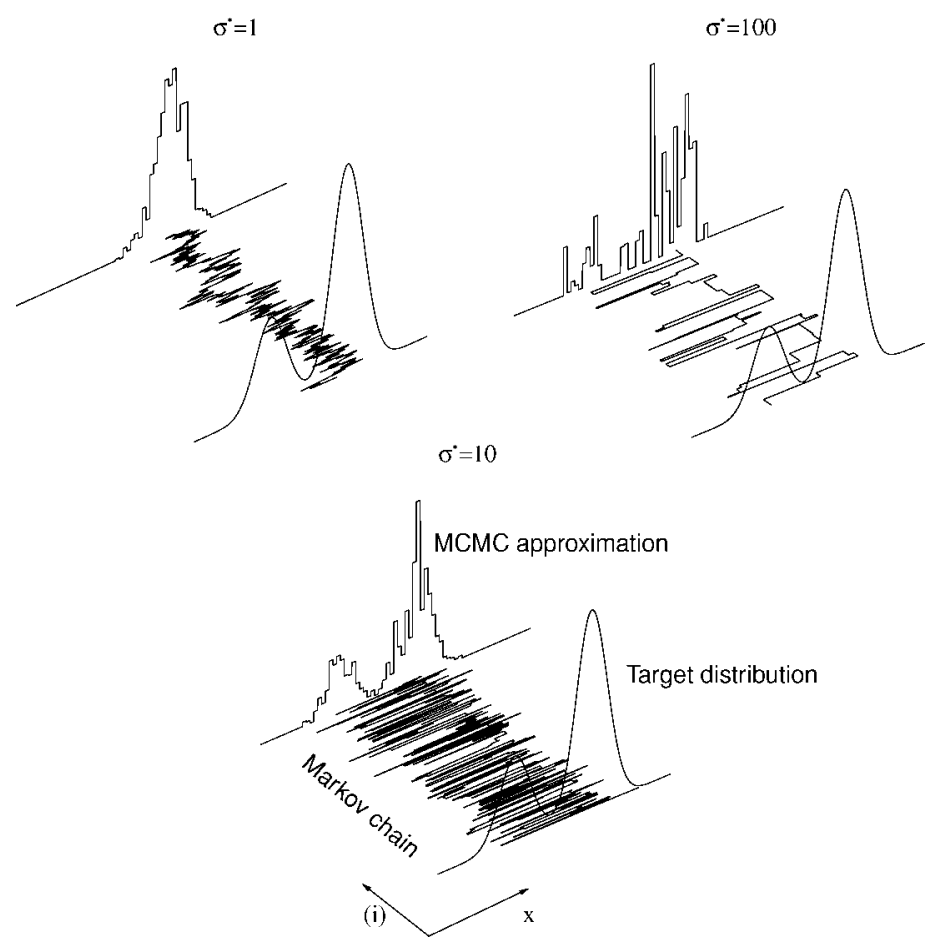

Figure 7. Approximations obtained using the MH algorithm with three Gaussian proposal distributions of different variances.

\subsection{Simulated annealing for global optimization}

Let us assume that instead of wanting to approximate $p(x)$, we want to find its global maximum. For example, if $p(x)$ is the likelihood or posterior distribution, we often want to compute the ML and maximum a posteriori (MAP) estimates. As mentioned earlier, we could run a Markov chain of invariant distribution $p(x)$ and estimate the global mode by

$$
\hat{x}=\underset{x^{(i)} ; i=1, \ldots, N}{\arg \max } p\left(x^{(i)}\right)
$$

This method is inefficient because the random samples only rarely come from the vicinity of the mode. Unless the distribution has large probability mass around the mode, computing resources will be wasted exploring areas of no interest. A more principled strategy is to adopt simulated annealing (Geman \& Geman, 1984; Kirkpatrick, Gelatt, \& Vecchi, 1983; Van Laarhoven \& Arts, 1987). This technique involves simulating a non-homogeneous Markov chain whose invariant distribution at iteration $i$ is no longer equal to $p(x)$, but to

$$
p_{i}(x) \propto p^{1 / T_{i}}(x)
$$


1. Initialise $x^{(0)}$ and set $T_{0}=1$.

2. For $i=0$ to $N-1$

- Sample $u \sim \mathcal{U}_{[0,1]}$.

- Sample $x^{\star} \sim q\left(x^{\star} \mid x^{(i)}\right)$.

- If $u<\mathcal{A}\left(x^{(i)}, x^{\star}\right)=\min \left\{1, \frac{p^{\frac{1}{T_{i}}}\left(x^{\star}\right) q\left(x^{(i)} \mid x^{\star}\right)}{p^{\frac{1}{T_{i}}}\left(x^{(i)}\right) q\left(x^{\star} \mid x^{(i)}\right)}\right\}$ $x^{(i+1)}=x^{\star}$

else

$x^{(i+1)}=x^{(i)}$

- Set $T_{i+1}$ according to a chosen cooling schedule.

Figure 8. General simulated annealing algorithm.
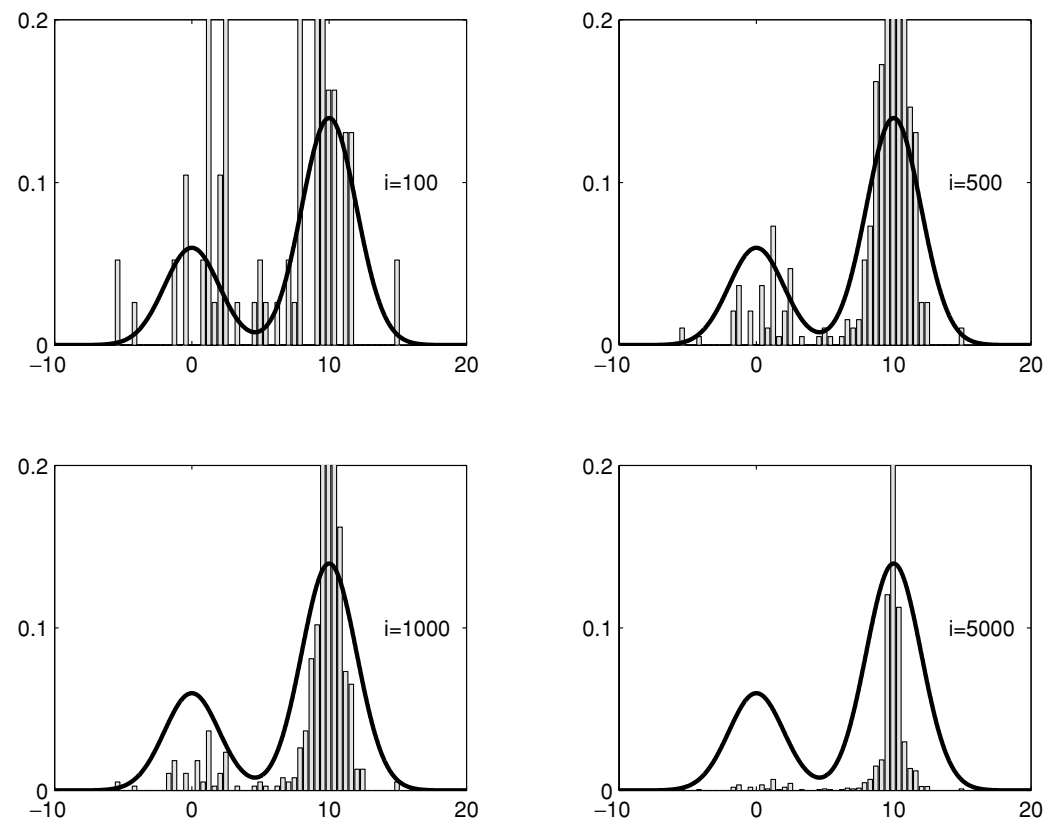

Figure 9. Discovering the modes of the target distribution with the simulated annealing algorithm.

where $T_{i}$ is a decreasing cooling schedule with $\lim _{i \rightarrow \infty} T_{i}=0$. The reason for doing this is that, under weak regularity assumptions on $p(x), p^{\infty}(x)$ is a probability density that concentrates itself on the set of global maxima of $p(x)$. The simulated annealing involves, therefore, just a minor modification of standard MCMC algorithms as shown in figure 8 . The results of applying annealing to the example of the previous section are shown in figure 9.

To obtain efficient annealed algorithms, it is again important to choose suitable proposal distributions and an appropriate cooling schedule. Many of the negative simulated annealing 
results reported in the literature often stem from poor proposal distribution design. In some complex variable and model selection scenarios arising in machine learning, one can even propose from complex reversible jump MCMC kernels (Section 3.7) within the annealing algorithm (Andrieu, de Freitas, \& Doucet, 2000a). If one defines a joint distribution over the parameter and model spaces, this technique can be used to search for the best model (according to MDL or AIC criteria) and ML parameter estimates simultaneously.

Most convergence results for simulated annealing typically state that if for a given $T_{i}$, the homogeneous Markov transition kernel mixes quickly enough, then convergence to the set of global maxima of $p(x)$ is ensured for a sequence $T_{i}=\left(C \ln \left(i+T_{0}\right)\right)^{-1}$, where $C$ and $T_{0}$ are problem-dependent. Most of the results have been obtained for finite spaces (Geman \& Geman, 1984; Van Laarhoven \& Arts, 1987) or compact continuous spaces (Haario \& Sacksman, 1991). Some results for non-compact spaces can be found in Andrieu, Breyer, and Doucet (1999).

\subsection{Mixtures and cycles of MCMC kernels}

A very powerful property of MCMC is that it is possible to combine several samplers into mixtures and cycles of the individual samplers (Tierney, 1994). If the transition kernels $K_{1}$ and $K_{2}$ have invariant distribution $p(\cdot)$ each, then the cycle hybrid kernel $K_{1} K_{2}$ and the mixture hybrid kernel $v K_{1}+(1-v) K_{2}$, for $0 \leq v \leq 1$, are also transition kernels with invariant distribution $p(\cdot)$.

Mixtures of kernels can incorporate global proposals to explore vast regions of the state space and local proposals to discover finer details of the target distribution (Andrieu, de Freitas, \& Doucet, 2000b; Andrieu \& Doucet, 1999; Robert \& Casella, 1999). This will be useful, for example, when the target distribution has many narrow peaks. Here, a global proposal locks into the peaks while a local proposal allows one to explore the space around each peak. For example, if we require a high-precision frequency detector, one can use the fast Fourier transform (FFT) as a global proposal and a random walk as local proposal (Andrieu \& Doucet, 1999). Similarly, in kernel regression and classification, one might want to have a global proposal that places the bases (kernels) at the locations of the input data and a local random walk proposal that perturbs these in order to obtain better fits (Andrieu, de Freitas, \& Doucet, 2000b). However, mixtures of kernels also play a big role in many other samplers, including the reversible jump MCMC algorithm (Section 3.7). The pseudo-code for a typical mixture of kernels is shown in figure 10 .

Cycles allow us to split a multivariate state vector into components (blocks) that can be updated separately. Typically the samplers will mix more quickly by blocking highly correlated variables. A block MCMC sampler, using $b_{j}$ to indicate the $j$-th block, $n_{b}$ to denote the number of blocks and $x_{-\left[b_{j}\right]}^{(i+1)} \triangleq\left\{x_{b_{1}}^{(i+1)}, x_{b_{2}}^{(i+1)}, \ldots, x_{b_{j-1}}^{(i+1)}, x_{b_{j+1}}^{(i)}, \ldots, x_{b_{n_{b}}}^{(i)}\right\}$, is shown in figure 11. The transition kernel for this algorithm is given by the following expression

$$
K_{\mathrm{MH}-\mathrm{Cycle}}\left(x^{(i+1)} \mid x^{(i)}\right)=\prod_{j=1}^{n_{b}} K_{\mathrm{MH}(\mathrm{j})}\left(x_{b_{j}}^{(i+1)} \mid x_{b_{j}}^{(i)}, x_{-\left[b_{j}\right]}^{(i+1)}\right)
$$

where $K_{\mathrm{MH}(\mathrm{j})}$ denotes the $j$-th $\mathrm{MH}$ algorithm in the cycle. 


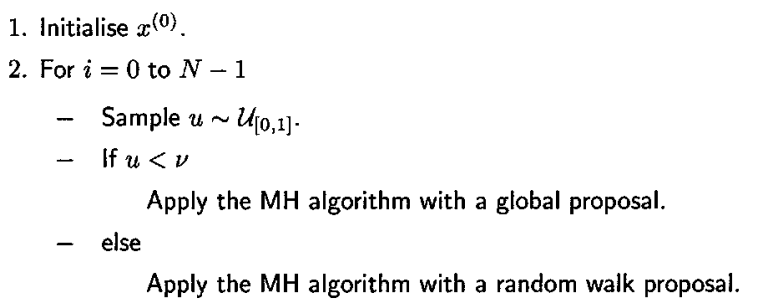

Figure 10. Typical mixture of MCMC kernels.

1. Initialise $x^{(0)}$.

2. For $i=0$ to $N-1$

- Sample the block $x_{b_{1}}^{(i+1)}$ according to an MH step with proposal distribution $q_{1}\left(x_{b_{1}}^{(i+1)} \mid x_{\cdots\left[b_{1}\right]}^{(i+1)}, x_{b_{1}}^{(i)}\right)$ and invariant distribution $p\left(x_{b_{1}}^{(i+1)} \mid x_{-\left[b_{1}\right]}^{(i+1)}\right)$.

- Sample the block $x_{b_{2}}^{(i+1)}$ according to an MH step with proposal distribution $q_{2}\left(x_{b_{2}}^{(i+1)} \mid x_{-\left[b_{2}\right]}^{(i+1)}, x_{b_{2}}^{(i)}\right)$ and invariant distribution $p\left(x_{b_{2}}^{(i+1)} \mid x_{-\left[b_{2}\right]}^{(i+1)}\right)$.

- Sample the block $x_{b_{n_{b}}}^{(i+1)}$ according to an MH step with proposal distribution $q_{n_{b}}\left(x_{b_{n_{b}}}^{(i+1)} \mid x_{-\left[b_{n_{b}}\right]}^{(i+1)}, x_{b_{n_{b}}}^{(i)}\right)$ and invariant distribution $p\left(x_{b_{n_{b}}}^{(i+1)} \mid x_{-\left[b_{n_{b}}\right]}^{(i+1)}\right)$.

Figure 11. Cycle of MCMC kernels-block MH algorithm.

Obviously, choosing the size of the blocks poses some trade-offs. If one samples the components of a multi-dimensional vector one-at-a-time, the chain may take a very long time to explore the target distribution. This problem gets worse as the correlation between the components increases. Alternatively, if one samples all the components together, then the probability of accepting this large move tends to be very low.

A popular cycle of MH kernels, known as Gibbs sampling (Geman \& Geman, 1984), is obtained when we adopt the full conditional distributions $p\left(x_{j} \mid x_{-j}\right)=p\left(x_{j} \mid x_{1}, \ldots, x_{j-1}\right.$, $x_{j+1}, \ldots, x_{n}$ ) as proposal distributions (for notational simplicity, we have replaced the index notation $b_{j}$ with $j$ ). The following section describes it in more detail.

\subsection{The Gibbs sampler}

Suppose we have an $n$-dimensional vector $x$ and the expressions for the full conditionals $p\left(x_{j} \mid x_{1}, \ldots, x_{j-1}, x_{j+1}, \ldots, x_{n}\right)$. In this case, it is often advantageous to use the following 
proposal distribution for $j=1, \ldots, n$

$$
q\left(x^{\star} \mid x^{(i)}\right)= \begin{cases}p\left(x_{j}^{\star} \mid x_{-j}^{(i)}\right) & \text { If } x_{-j}^{\star}=x_{-j}^{(i)} \\ 0 & \text { Otherwise. }\end{cases}
$$

The corresponding acceptance probability is:

$$
\begin{aligned}
\mathcal{A}\left(x^{(i)}, x^{\star}\right) & =\min \left\{1, \frac{p\left(x^{\star}\right) q\left(x^{(i)} \mid x^{\star}\right)}{p\left(x^{(i)}\right) q\left(x^{\star} \mid x^{(i)}\right)}\right\} \\
& =\min \left\{1, \frac{p\left(x^{\star}\right) p\left(x_{j}^{(i)} \mid x_{-j}^{(i)}\right)}{p\left(x^{(i)}\right) p\left(x_{j}^{\star} \mid x_{-j}^{\star}\right)}\right\} \\
& =\min \left\{1, \frac{p\left(x_{-j}^{\star}\right)}{p\left(x_{-j}^{(i)}\right)}\right\}=1 .
\end{aligned}
$$

That is, the acceptance probability for each proposal is one and, hence, the deterministic scan Gibbs sampler algorithm is often presented as shown in figure 12.

Since the Gibbs sampler can be viewed as a special case of the MH algorithm, it is possible to introduce MH steps into the Gibbs sampler. That is, when the full conditionals are available and belong to the family of standard distributions (Gamma, Gaussian, etc.), we will draw the new samples directly. Otherwise, we can draw samples with MH steps embedded within the Gibbs algorithm. For $n=2$, the Gibbs sampler is also known as the data augmentation algorithm, which is closely related to the expectation maximisation (EM) algorithm (Dempster, Laird, \& Rubin, 1977; Tanner \& Wong, 1987).

Directed acyclic graphs (DAGS) are one of the best known application areas for Gibbs sampling (Pearl, 1987). Here, a large-dimensional joint distribution is factored into a directed graph that encodes the conditional independencies in the model. In particular, if $x_{p a(j)}$

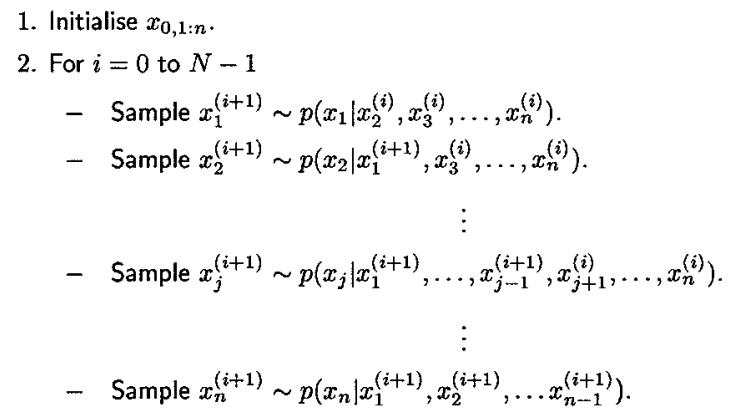

Figure 12. Gibbs sampler. 
denotes the parent nodes of node $x_{j}$, we have

$$
p(x)=\prod_{j} p\left(x_{j} \mid x_{p a(j)}\right) .
$$

It follows that the full conditionals simplify as follows

$$
p\left(x_{j} \mid x_{-j}\right)=p\left(x_{j} \mid x_{p a(j)}\right) \prod_{k \in c h(j)} p\left(x_{k} \mid x_{p a(k)}\right)
$$

where $\operatorname{ch}(j)$ denotes the children nodes of $x_{j}$. That is, we only need to take into account the parents, the children and the children's parents. This set of variables is known as the Markov blanket of $x_{j}$. This technique forms the basis of the popular software package for Bayesian updating with Gibbs sampling (BUGS) (Gilks, Thomas, \& Spiegelhalter, 1994). Sampling from the full conditionals, with the Gibbs sampler, lends itself naturally to the construction of general purpose MCMC software. It is sometimes convenient to block some of the variables to improve mixing (Jensen, Kong, \& Kjærulff, 1995; Wilkinson \& Yeung, 2002).

\subsection{Monte Carlo EM}

The EM algorithm (Baum et al., 1970; Dempster, Laird, \& Rubin, 1977) is a standard algorithm for ML and MAP point estimation. If $\mathcal{X}$ contains visible and hidden variables $x=\left\{x_{v}, x_{h}\right\}$, then a local maximum of the likelihood $p\left(x_{v} \mid \theta\right)$ given the parameters $\theta$ can be found by iterating the following two steps:

1. E step. Compute the expected value of the complete log-likelihood function with respect to the distribution of the hidden variables

$$
Q(\theta)=\int_{\mathcal{X}_{h}} \log \left(p\left(x_{h}, x_{v} \mid \theta\right)\right) p\left(x_{h} \mid x_{v}, \theta^{\text {(old })}\right) d x_{h},
$$

where $\theta^{\text {(old) }}$ refers to the value of the parameters at the previous time step.

2. M step. Perform the following maximisation $\theta^{(\text {new })}=\arg \max _{\theta} Q(\theta)$.

In many practical situations, the expectation in the $\mathrm{E}$ step is either a sum with an exponentially large number of summands or an intractable integral (Ghahramani, 1995; Ghahramani \& Jordan, 1995; McCulloch, 1994; Pasula et al., 1999; Utsugi, 2001); see also Dellaert et al. (this issue). A solution is to introduce MCMC to sample from $p\left(x_{h} \mid x_{v}, \theta^{\text {(old) }}\right)$ and replace the expectation in the E step with a small sum over the samples, as shown in figure 13. Convergence of this algorithm is discussed in Sherman, Ho, and Dalal (1999), while Levine and Casella (2001) is a good recent review.

To improve the convergence behaviour of EM, namely to escape low local minima and saddle points, various authors have proposed stochastic approaches that rely on sampling from $p\left(x_{h} \mid x_{v}, \theta^{\text {(old) }}\right)$ in the $\mathrm{E}$ step and then performing the $\mathrm{M}$ step using these samples. 
1. Initialise $\left(x_{h}^{(0)}, \theta^{(0)}\right)$ and set $i=0$.

2. Iteration $i$ of EM

- Sample $\left\{x_{h}^{(j)}\right\}_{j=1}^{N_{i}}$ with any suitable MCMC algorithm. For example, one could use an MH algorithm with acceptance probability

$$
\mathcal{A}=\min \left\{1, \frac{p\left(x_{v} \mid x_{h}^{\star}, \theta^{(i-1)}\right) p\left(x_{h}^{\star} \mid \theta^{(i-1)}\right) q\left(x_{h}^{(j)} \mid x_{h}^{\star}\right)}{p\left(x_{v} \mid x_{h}^{(j)}, \theta^{(i-1)}\right) p\left(x_{h}^{(j)} \mid \theta^{(i-1)}\right) q\left(x_{h}^{\star} \mid x_{h}^{(j)}\right)}\right\}
$$

- E step: Compute

$$
\widehat{Q}(\theta)=\frac{1}{N_{i}} \sum_{j=1}^{N_{i}} \log p\left(x_{h}^{(j)}, x_{v} \mid \theta\right)
$$

- M step: Maximise $\theta^{(i)}=\underset{\theta}{\arg \max } \widehat{Q}(\theta)$.

3. $i \leftarrow i+1$ and go to 2 .

Figure 13. MCMC-EM algorithm.

The method is known as stochastic EM (SEM) when we draw only one sample (Celeux \& Diebolt, 1985) and Monte Carlo EM (MCEM) when several samples are drawn (Wei $\&$ Tanner, 1990). There are several annealed variants (such as SAEM) that become more deterministic as the number of iterations increases (Celeux \& Diebolt, 1992). The are also very efficient algorithms for marginal MAP estimation (SAME) (Doucet, Godsill, \& Robert, 2000). One wishes sometimes that Metropolis had succeeded in stopping the proliferation of acronyms!

\subsection{Auxiliary variable samplers}

It is often easier to sample from an augmented distribution $p(x, u)$, where $u$ is an auxiliary variable, than from $p(x)$. Then, it is possible to obtain marginal samples $x^{(i)}$ by sampling $\left(x^{(i)}, u^{(i)}\right)$ according to $p(x, u)$ and, subsequently, ignoring the samples $u^{(i)}$. This very useful idea was proposed in the physics literature (Swendsen \& Wang, 1987). Here, we will focus on two well-known examples of auxiliary variable methods, namely hybrid Monte Carlo and slice sampling.

3.6.1. Hybrid Monte Carlo. Hybrid Monte Carlo (HMC) is an MCMC algorithm that incorporates information about the gradient of the target distribution to improve mixing in high dimensions. We describe here the "leapfrog" HMC algorithm outlined in Duane et al. (1987) and Neal (1996) focusing on the algorithmic details and not on the statistical mechanics motivation. Assume that $p(x)$ is differentiable and everywhere strictly positive. At each iteration of the HMC algorithm, one takes a predetermined number $(L)$ of deterministic steps using information about the gradient of $p(x)$. To explain this in more detail, we first need to introduce a set of auxiliary "momentum" variables $u \in \mathbb{R}^{n_{x}}$ and define the 


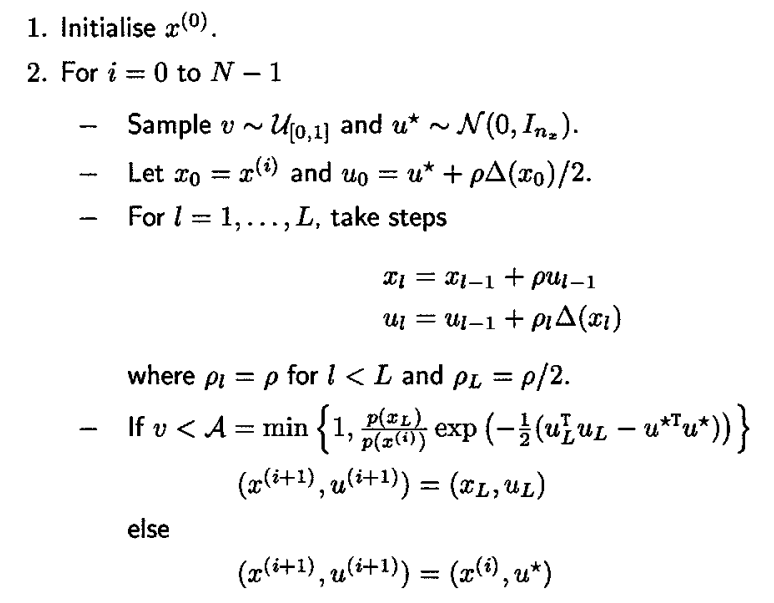

Figure 14. Hybrid Monte Carlo.

extended target density

$$
p(x, u)=p(x) \mathcal{N}\left(u ; 0, I_{n_{x}}\right) .
$$

Next, we need to introduce the $n_{x}$-dimensional gradient vector $\Delta(x) \triangleq \partial \log p(x) / \partial x$ and a fixed step-size parameter $\rho>0$.

In the HMC algorithm, we draw a new sample according to $p(x, u)$ by starting with the previous value of $x$ and generating a Gaussian random variable $u$. We then take $\mathrm{L}$ "frog leaps" in $u$ and $x$. The values of $u$ and $x$ at the last leap are the proposal candidates in the MH algorithm with target density $p(x, u)$. Marginal samples from $p(x)$ are obtained by simply ignoring $u$. Given $\left(x^{(i-1)}, u^{(i-1)}\right)$, the algorithm proceeds as illustrated in figure 14 .

When only one deterministic step is used, i.e. $L=1$, one obtains the Langevin algorithm, which is a discrete time approximation of a Langevin diffusion process. The Langevin algorithm is a special case of $\mathrm{MH}$ where the candidate satisfies

$$
x^{\star}=x_{0}+\rho u_{0}=x^{(i-1)}+\rho\left(u^{\star}+\rho \Delta\left(x^{(i-1)}\right) / 2\right)
$$

with $u^{\star} \sim \mathcal{N}\left(0, I_{n_{x}}\right)$.

The choice of the parameters $L$ and $\rho$ poses simulation tradeoffs. Large values of $\rho$ result in low acceptance rates, while small values require many leapfrog steps (expensive computations of the gradient) to move between two nearby states. Choosing $L$ is equally problematic as we want it to be large to generate candidates far from the initial state, but this can result in many expensive computations. HMC, therefore, requires careful tuning of the proposal distribution. It is more efficient, in practice, to allow a different step size $\rho$ for each of the coordinates of $x$ (Ishwaran, 1999). 
3.6.2. The slice sampler. The slice sampler (Damien, Wakefield, \& Walker, 1999; Higdon, 1998; Wakefield, Gelfand, \& Smith, 1991) is a general version of the Gibbs sampler. The basic idea of the slice sampler is to introduce an auxiliary variable $u \in \mathbb{R}$ and construct an extended target distribution $p^{\star}(x, u)$, such that

$$
p^{\star}(x, u)= \begin{cases}1 & \text { if } 0 \leq u \leq p(x) \\ 0 & \text { otherwise }\end{cases}
$$

It is then straightforward to check that

$$
\int p^{\star}(x, u) d u=\int_{0}^{p(x)} d u=p(x) .
$$

Hence, to sample from $p(x)$ one can sample from $p^{\star}(x, u)$ and then ignore $u$. The full conditionals are of this augmented model are

$$
\begin{aligned}
& p(u \mid x)=\mathcal{U}_{[0, p(x)]}(u) \\
& p(x \mid u)=\mathcal{U}_{A}(x)
\end{aligned}
$$

where $A=\{x ; p(x) \geq u\}$. If $A$ is easy to identify then the algorithm is straightforward to implement, as shown in figure 15 .

It can be difficult to identify $A$. It is then worth introducing several auxiliary variables (Damien, Wakefield, \& Walker, 1999; Higdon, 1998). For example assume that

$$
p(x) \propto \prod_{l=1}^{L} f_{l}(x),
$$

where the $f_{l}(\cdot)$ 's are positive functions, not necessarily densities. Let us introduce $L$ auxiliary variables $\left(u_{1}, \ldots, u_{L}\right)$ and define

$$
p^{\star}\left(x, u_{1}, \ldots, u_{L}\right) \propto \prod_{l=1}^{L} \mathbb{I}_{\left[0, f_{l}(x)\right]}\left(u_{l}\right) .
$$

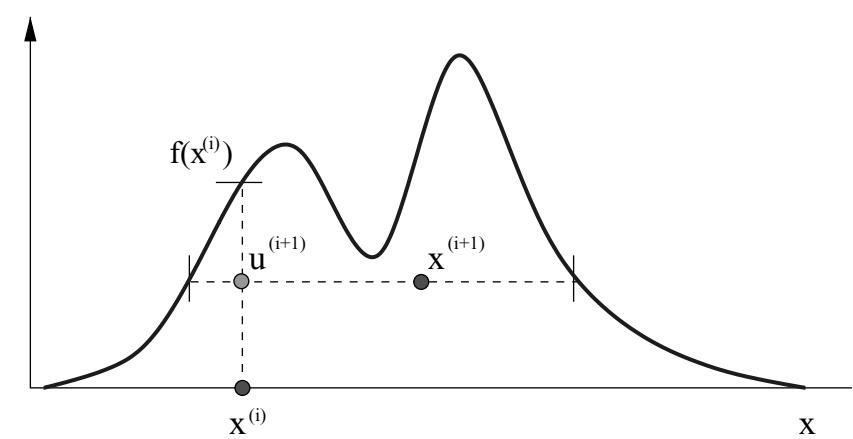

Figure 15. Slice sampling: given a previous sample, we sample a uniform variable $u^{(i+1)}$ between 0 and $f\left(x^{(i)}\right)$. One then samples $x^{(i+1)}$ in the interval where $f(x) \geq u^{(i+1)}$. 


$$
\begin{aligned}
& \text { 1. For } l=1, \ldots, L \\
& \quad \text { - Sample } u_{l}^{(i)} \sim \mathcal{U}_{\left[0, f_{l}\left(x^{(i-1)}\right)\right]}{ }^{\left(u_{l}\right)} \\
& \text { 2. Sample } x^{(i)} \sim \mathcal{U}_{A^{(i)}}(x) \text { where } A^{(i)}=\left\{x ; f_{l}(x) \geq u_{l}^{(i)}, l=1, \ldots, L\right\} \text {. }
\end{aligned}
$$

Figure 16. Slice sampler.

Then one can also check that $\int p^{\star}\left(x, u_{1}, \ldots, u_{L}\right) d u_{1} \ldots d u_{L}=p(x)$ as

$$
\int p^{\star}\left(x, u_{1}, \ldots, u_{L}\right) d u_{1} \ldots d u_{L} \propto \int \prod_{l=1}^{L} \mathbb{I}_{\left[0, f_{l}(x)\right]}\left(u_{l}\right) d u_{1} \ldots d u_{L}=\prod_{l=1}^{L} f_{l}(x) .
$$

The slice sampler to sample from $p^{\star}\left(x, u_{1}, \ldots, u_{L}\right)$ proceeds as shown in figure 16. Algorithmic improvements and convergence results are presented in Mira (1999) and Neal (2000).

\subsection{Reversible jump MCMC}

In this section, we attack the more complex problem of model selection. Typical examples include estimating the number of neurons in a neural network (Andrieu, de Freitas, \& Doucet, 2001a; Holmes \& Mallick, 1998; Rios Insua \& Müller, 1998), the number of splines in a multivariate adaptive splines regression (MARS) model (Holmes \& Denison, this issue), the number of sinusoids in a noisy signal (Andrieu \& Doucet, 1999), the number of lags in an autoregressive process (Troughton \& Godsill, 1998), the number of components in a mixture (Richardson \& Green, 1997), the number of levels in a changepoint process (Green, 1995), the number of components in a mixture of factor analysers (Fokoue \& Titterington, this issue), the appropriate structure of a graphical model (Friedman \& Koller, 2001; Giudici \& Castelo, this issue) or the best set of input variables (Lee, this issue).

Given a family of $M$ models $\left\{\mathcal{M}_{m} ; m=1, \ldots, N\right\}$, we will focus on constructing ergodic Markov chains admitting $p\left(m, x_{m}\right)$ as the invariant distribution. For simplicity, we avoid the treatment of nonparametric model averaging techniques; see for example (Escobar \& West, 1995; Green \& Richardson, 2000).

Up to this section, we have been comparing densities in the acceptance ratio. However, if we are carrying out model selection, then comparing the densities of objects in different dimensions has no meaning. It is like trying to compare spheres with circles. Instead, we have to be more formal and compare distributions $P(d x)=\operatorname{Pr}(x \in d x)$ under a common measure of volume. The distribution $P(d x)$ will be assumed to admit a density $p(x)$ with respect to a measure of interest, e.g. Lebesgue in the continuous case: $P(d x)=p(x) d x$. The acceptance ratio will now include the ratio of the densities and the ratio of the measures (Radon Nikodym derivative). The latter gives rise to a Jacobian term. To compare densities point-wise, we need, therefore, to map the two models to a common dimension as illustrated in figure 17. 
Univariate density

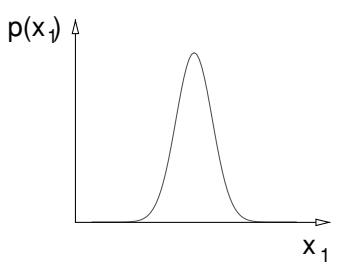

Bivariate density

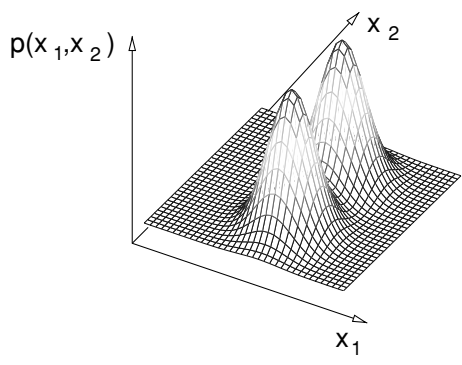

Uniformly expanded density

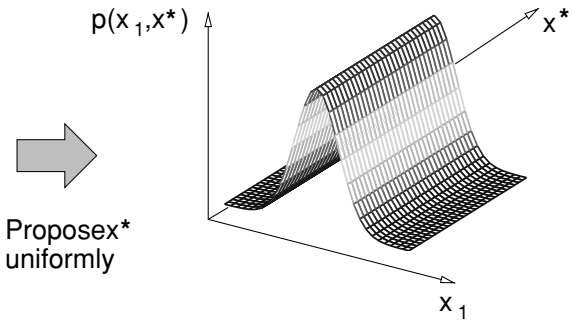

Figure 17. To compare a 1D model against a 2D model, we first have to map the first model so that both models have common measure (area in this case).

The parameters $x_{m} \in \mathcal{X}_{m}$ (e.g. $\mathcal{X}_{m}=\mathbb{R}^{n_{m}}$ ) are model dependent. Hence, to find the right model and parameters we could sample over the model indicator and the product space $\prod_{m=1}^{M} \mathcal{X}_{m}$ (Carlin \& Chib, 1995). Recently, Green introduced a strategy that avoids this expensive search over the full product space (Green, 1995). In particular one samples on a much smaller union space $\mathcal{X} \triangleq \bigcup_{m=1}^{M}\{m\} \times \mathcal{X}_{m}$. The full target distribution defined in this space is given by

$$
p(k, d x)=\sum_{m=1}^{M} p\left(m, d x_{m}\right) \mathbb{I}_{\{m\} \times \mathcal{X}_{m}}(k, x) .
$$

That is, the probability of $k$ being equal to $m$ and $x$ belonging to an infinitesimal set centred around $x_{m}$ is $p\left(m, d x_{m}\right)$. By marginalisation, we obtain the probability of being in subspace $\mathcal{X}_{m}$.

Green's method allows the sampler to jump between the different subspaces. To ensure a common measure, it requires the extension of each pair of communicating spaces, $\mathcal{X}_{m}$ and $\mathcal{X}_{n}$, to $\overline{\mathcal{X}}_{m, n} \triangleq \mathcal{X}_{m} \times \mathcal{U}_{m, n}$ and $\overline{\mathcal{X}}_{n, m} \triangleq \mathcal{X}_{n} \times \mathcal{U}_{n, m}$. It also requires the definition of a deterministic, differentiable, invertible dimension matching function $f_{n \rightarrow m}$ between $\overline{\mathcal{X}}_{m, n}$ and $\overline{\mathcal{X}}_{n, m}$,

$$
\left(x_{m}, u_{m, n}\right)=f_{n \rightarrow m}\left(x_{n}, u_{n, m}\right)=\left(f_{n \rightarrow m}^{x}\left(x_{n}, u_{n, m}\right), f_{n \rightarrow m}^{u}\left(x_{n}, u_{n, m}\right)\right) .
$$


We define $f_{m \rightarrow n}$ such that $f_{m \rightarrow n}\left(f_{n \rightarrow m}\left(x_{n}, u_{n, m}\right)\right)=\left(x_{n}, u_{n, m}\right)$. The choice of the extended spaces, deterministic transformation $f_{m \rightarrow n}$ and proposal distributions for $q_{n \rightarrow m}\left(\cdot \mid n, x_{n}\right)$ and $q_{m \rightarrow n}\left(\cdot \mid m, x_{m}\right)$ is problem dependent and needs to be addressed on a case by case basis.

If the current state of the chain is $\left(n, x_{n}\right)$, we move to $\left(m, x_{m}\right)$ by generating $u_{n, m} \sim$ $q_{n \rightarrow m}\left(\cdot \mid n, x_{n}\right)$, ensuring that we have reversibility $\left(x_{m}, u_{m, n}\right)=f_{n \rightarrow m}\left(x_{n}, u_{n, m}\right)$, and accepting the move according to the probability ratio

$$
\mathcal{A}_{n \rightarrow m}=\min \left\{1, \frac{p\left(m, x_{m}^{\star}\right)}{p\left(n, x_{n}\right)} \times \frac{q(n \mid m)}{q(m \mid n)} \times \frac{q_{m \rightarrow n}\left(u_{m, n} \mid m, x_{m}^{\star}\right)}{q_{n \rightarrow m}\left(u_{n, m} \mid n, x_{n}\right)} \times \mathcal{J}_{f_{n \rightarrow m}}\right\},
$$

where $x_{m}^{\star}=f_{n \rightarrow m}^{x}\left(x_{n}, u_{n, m}\right)$ and $\mathcal{J}_{f_{n \rightarrow m}}$ is the Jacobian of the transformation $f_{n \rightarrow m}$ (when only continuous variables are involved in the transformation)

$$
\mathcal{J}_{f_{m \rightarrow n}}=\left|\operatorname{det} \frac{\partial f_{n \rightarrow m}\left(x_{m}, u_{m, n}\right)}{\partial\left(x_{m}, u_{m, n}\right)}\right| .
$$

To illustrate this, assume that we are concerned with sampling the locations $\mu$ and number $k$ of components of a mixture. For example we might want to estimate the locations and number of basis functions in kernel regression and classification, the number of mixture components in a finite mixture model, or the location and number of segments in a segmentation problem. Here, we could define a merge move that combines two nearby components and a split move that breaks a component into two nearby ones. The merge move involves randomly selecting a component $\left(\mu_{1}\right)$ and then combining it with its closest neighbour $\left(\mu_{2}\right)$ into a single component $\mu$, whose new location is

$$
\mu=\frac{\mu_{1}+\mu_{2}}{2}
$$

The corresponding split move that guarantees reversibility, involves splitting a randomly chosen component as follows

$$
\begin{aligned}
& \mu_{1}=\mu-u_{n, m} \beta \\
& \mu_{2}=\mu+u_{n, m} \beta
\end{aligned}
$$

where $\beta$ is a simulation parameter and, for example, $u_{n, m} \sim \mathcal{U}_{[0,1]}$. Note that to ensure reversibility, we only perform the merge move if $\left\|\mu_{1}-\mu_{2}\right\|<2 \beta$. The acceptance ratio for the split move is

$$
\mathcal{A}_{\text {split }}=\min \left\{1, \frac{p\left(k+1, \mu_{k+1}\right)}{p\left(k, \mu_{k}\right)} \times \frac{\frac{1}{k+1}}{\frac{1}{k}} \times \frac{1}{p\left(u_{n, m}\right)} \times \mathcal{J}_{\text {split }}\right\},
$$

where $1 / k$ denotes the probability of choosing, uniformly at random, one of the $k$ components. The Jacobian is

$$
\mathcal{J}_{\text {split }}=\left|\frac{\partial\left(\mu_{1}, \mu_{2}\right)}{\partial\left(\mu, u_{n, m}\right)}\right|=\left|\begin{array}{cc}
1 & 1 \\
-\beta & \beta
\end{array}\right|=2 \beta .
$$




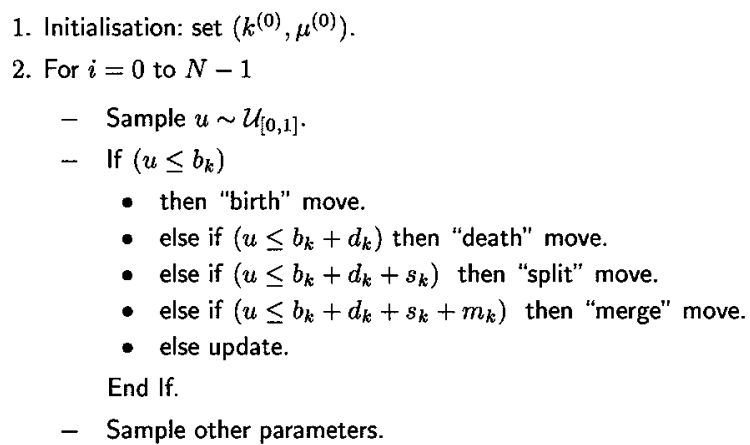

Figure 18. Generic reversible jump MCMC.

Similarly, for the merge move, we have

$$
\mathcal{A}_{\text {merge }}=\min \left\{1, \frac{p\left(k-1, \mu_{k-1}\right)}{p\left(k, \mu_{k}\right)} \times \frac{\frac{1}{k-1}}{\frac{1}{k}} \times \mathcal{J}_{\text {merge }}\right\} \text {, }
$$

where $\mathcal{J}_{\text {merge }}=1 / 2 \beta$.

Reversible jump is a mixture of MCMC kernels (moves). In addition, to the split and merge moves, we could have other moves such as birth of a component, death of a component and a simple update of the locations. The various moves are carried out according to the mixture probabilities $\left(b_{k}, d_{k}, m_{k}, s_{k}, u_{k}\right)$, as shown in figure 18 . In fact, it is the flexibility of including so many possible moves that can make reversible jump a more powerful model selection strategy than schemes based on model selection using a mixture indicator or diffusion processes using only birth and death moves (Stephens, 1997). However, the problem with reversible jump MCMC is that engineering reversible moves is a very tricky, time-consuming task.

\section{The MCMC frontiers}

\subsection{Convergence and perfect sampling}

Determining the length of the Markov chain is a difficult task. In practice, one often discards an initial set of samples (burn-in) to avoid starting biases. In addition, one can apply several graphical and statistical tests to assess, roughly, if the chain has stabilised (Robert \& Casella, 1999, ch. 8). In general, none of these tests provide entirely satisfactory diagnostics.

Several theoreticians have tried to bound the mixing time; that is, the minimum number of steps required for the distribution of the Markov chain $K$ to be close to the target $p(x)$. (Here, we present a, by no means exhaustive, summary of some of the available results.) If 
we measure closeness with the total variation norm $\Delta_{x}(t)$, where

$$
\Delta_{x}(t)=\left\|K^{(t)}(\cdot \mid x)-p(\cdot)\right\|=\frac{1}{2} \int\left(K^{(t)}(y \mid x)-p(y)\right) d y,
$$

then the mixing time is

$$
\tau_{x}(\epsilon)=\min \left\{t: \Delta_{x}\left(t^{\prime}\right) \leq \epsilon \text { for all } t^{\prime} \geq t\right\} .
$$

If the state space $\mathcal{X}$ is finite and reversibility holds true, then the transition operator $K\left(K f(x)=\sum K(y \mid x) f(y)\right)$ is self adjoint on $L_{2}(p)$. That is,

$$
\langle K f \mid g\rangle=\langle f \mid K g\rangle,
$$

where $f$ and $g$ are real functions and we have used the bra-ket notation for the inner product $\langle f \mid g\rangle=\sum f(x) g(x) p(x)$. This implies that $K$ has real eigenvalues

$$
1=\lambda_{1}>\lambda_{2} \geq \lambda_{3} \geq \cdots \geq \lambda_{|\mathcal{X}|}>-1
$$

and an orthonormal basis of real eigenfunctions $f_{i}$, such that $K f_{i}=\lambda_{i} f_{i}$. This spectral decomposition and the Cauchy-Schwartz inequality allow us to obtain a bound on the total variation norm

$$
\Delta_{x}(t) \leq \frac{1}{2 \sqrt{p(x)}} \lambda_{\star}^{t},
$$

where $\lambda_{\star}=\max \left(\lambda_{2},\left|\lambda_{|\mathcal{X}|}\right|\right)$ (Diaconis \& Saloff-Coste, 1998; Jerrum \& Sinclair, 1996). This classical result give us a geometric convergence rate in terms of eigenvalues. Geometric bounds have also been obtained in general state spaces using the tools of regeneration and Lyapunov-Foster conditions (Meyn \& Tweedie, 1993).

The next logical step is to bound the second eigenvalue. There are several inequalities (Cheeger, Poincaré, Nash) from differential geometry that allows us to obtain these bounds (Diaconis \& Saloff-Coste, 1998). For example, one could use Cheeger's inequality to obtain the following bound

$$
1-2 \Phi \leq \lambda_{2} \leq 1-\frac{\Phi^{2}}{2}
$$

where $\Phi$ is the conductance of the Markov chain

$$
\Phi=\min _{0<p(S)<1 / 2 ; S \subset \mathcal{X}} \frac{\sum_{x \in S, y \in S^{c}} p(x) K(y \mid x)}{p(S)}
$$

Intuitively, one can interpret this quantity as the readiness of the chain to escape from any small region $S$ of the state space and, hence, make rapid progress towards equilibrium (Jerrum \& Sinclair, 1996). 
These mathematical tools have been applied to show that simple MCMC algorithms (mostly Metropolis) run in time that is polynomial in the dimension $d$ of the state space, thereby escaping the exponential curse of dimensionality. Polynomial time sampling algorithms have been obtained in the following important scenarios:

1. Computing the volume of a convex body in $d$ dimensions, where $d$ is large (Dyer, Frieze, \& Kannan, 1991).

2. Sampling from log-concave distributions (Applegate \& Kannan, 1991).

3. Sampling from truncated multivariate Gaussians (Kannan \& Li, 1996).

4. Computing the permanent of a matrix (Jerrum, Sinclair, \& Vigoda, 2000).

The last problem is equivalent to sampling matchings from a bipartite graph; a problem that manifests itself in many ways in machine learning (e.g., stereo matching and data association).

Although the theoretical results are still far from the practice of MCMC, they will eventually provide better guidelines on how to design and choose algorithms. Already, some results tell us, for example, that it is not wise to use the independent Metropolis sampler in high dimensions (Mengersen \& Tweedie, 1996).

A remarkable recent breakthrough was the development of algorithms for perfect sampling. These algorithms are guaranteed to give us an independent sample from $p(x)$ under certain restrictions. The two major players are coupling from the past (Propp \& Wilson, 1998) and Fill's algorithm (Fill, 1998). From a practical point of view, these algorithms are still limited and, in many cases, computationally inefficient. However, some steps are being taken towards obtaining more general perfect samplers; for example perfect slice samplers (Casella et al., 1999).

\subsection{Adaptive MCMC}

If we look at the chain on the top right of figure 7, we notice that the chain stays at each state for a long time. This tells us that we should reduce the variance of the proposal distribution. Ideally, one would like to automate this process of choosing the proposal distribution as much as possible. That is, one should use the information in the samples to update the parameters of the proposal distribution so as to obtain a distribution that is either closer to the target distribution, that ensures a suitable acceptance rate, or that minimises the variance of the estimator of interest. However, one should not allow adaptation to take place infinitely often in a naive way because this can disturb the stationary distribution. This problem arises because by using the past information infinitely often, we violate the Markov property of the transition kernel. That is, $p\left(x^{(i)} \mid x^{(0)}, x^{(1)}, \ldots, x^{(i-1)}\right)$ no longer simplifies to $p\left(x^{(i)} \mid x^{(i-1)}\right)$. In particular, Gelfand and Sahu (1994) present a pathological example, where the stationary distribution is disturbed despite the fact that each participating kernel has the same stationary distribution.

To avoid this problem, one could carry out adaptation only during an initial fixed number of steps, and then use standard MCMC simulation to ensure convergence to the right distribution. Two methods for doing this are presented in Gelfand and Sahu (1994). The first is based on the idea of running several chains in parallel and using sampling-importance resampling 
(Rubin, 1988) to multiply the kernels that are doing well and suppress the others. In this approach, one uses an approximation to the marginal density of the chain as proposal. The second method simply involves monitoring the transition kernel and changing one of its components (for example the proposal distribution) so as to improve mixing. A similar method that guarantees a particular acceptance rate is discussed in Browne and Draper (2000).

There are, however, a few adaptive MCMC methods that allow one to perform adaptation continuously without disturbing the Markov property, including delayed rejection (Tierney \& Mira, 1999), parallel chains (Gilks \& Roberts, 1996) and regeneration (Gilks, Roberts, \& Sahu, 1998; Mykland, Tierney, \& Yu, 1995). These methods are, unfortunately, inefficient in many ways and much more research is required in this exciting area.

\subsection{Sequential Monte Carlo and particle filters}

Sequential Monte Carlo (SMC) methods allow us to carry out on-line approximation of probability distributions using samples (particles). They are very useful in scenarios involving real-time signal processing, where data arrival is inherently sequential. Furthermore, one might wish to adopt a sequential processing strategy to deal with non-stationarity in signals, so that information from the recent past is given greater weighting than information from the distant past. Computational simplicity in the form of not having to store all the data might also constitute an additional motivating factor for these methods.

In the SMC setting, we assume that we have an initial distribution, a dynamic model and measurement model

$$
\begin{aligned}
p\left(x_{0}\right) & \\
p\left(x_{t} \mid x_{0: t-1}, y_{1: t-1}\right) & \text { for } t \geq 1 \\
p\left(y_{t} \mid x_{0: t}, y_{1: t-1}\right) & \text { for } t \geq 1
\end{aligned}
$$

We denote by $x_{0: t} \triangleq\left\{x_{0}, \ldots, x_{t}\right\}$ and $y_{1: t} \triangleq\left\{y_{1}, \ldots, y_{t}\right\}$, respectively, the states and the observations up to time $t$. Note that we could assume Markov transitions and conditional independence to simplify the model; $p\left(x_{t} \mid x_{0: t-1}, y_{1: t-1}\right)=p\left(x_{t} \mid x_{t-1}\right)$ and $p\left(y_{t} \mid x_{0: t}, y_{1: t-1}\right)=$ $p\left(y_{t} \mid x_{t}\right)$. However, this assumption is not necessary in the SMC framework.

Our aim is to estimate recursively in time the posterior $p\left(x_{0: t} \mid y_{1: t}\right)$ and its associated features including the marginal distribution $p\left(x_{t} \mid y_{1: t}\right)$, known as the filtering distribution, and the expectations

$$
I\left(f_{t}\right)=\mathbb{E}_{p\left(x_{0: t} \mid y_{1: t}\right)}\left[f_{t}\left(x_{0: t}\right)\right]
$$

A generic SMC algorithm is depicted in figure 19. Given $N$ particles $\left\{x_{0: t-1}^{(i)}\right\}_{i=1}^{N}$ at time $t-1$, approximately distributed according to the distribution $p\left(x_{0: t-1} \mid y_{1: t-1}\right)$, SMC methods allow us to compute $N$ particles $\left\{x_{0: t}^{(i)}\right\}_{i=1}^{N}$ approximately distributed according to the posterior $p\left(x_{0: t} \mid y_{1: t}\right)$, at time $t$. Since we cannot sample from the posterior directly, the SMC update is accomplished by introducing an appropriate importance proposal distribution $q\left(x_{0: t}\right)$ from which we can obtain samples. The samples are then appropriately weighted. 


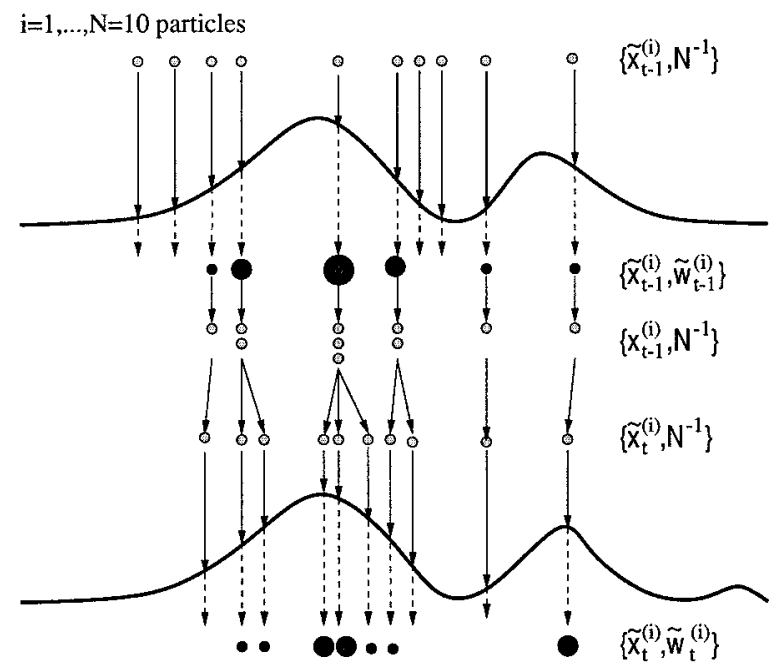

Figure 19. In this example, the bootstrap filter starts at time $t-1$ with an unweighted measure $\left\{\tilde{\mathbf{x}}_{t-1}^{(i)}, N^{-1}\right\}$, which provides an approximation of $p\left(\mathbf{x}_{t-1} \mid y_{1: t-2}\right)$. For each particle we compute the importance weights using the information at time $t-1$. This results in the weighted measure $\left\{\tilde{\mathbf{x}}_{t-1}^{(i)}, \tilde{w}_{t-1}^{(i)}\right\}$, which yields an approximation $p\left(\mathbf{x}_{t-1} \mid y_{1: t-1}\right)$. Subsequently, the resampling step selects only the "fittest" particles to obtain the unweighted measure $\left\{\mathbf{x}_{t-1}^{(i)}, N^{-1}\right\}$, which is still an approximation of $p\left(\mathbf{x}_{t-1} \mid y_{1: t-1}\right)$. Finally, the sampling (prediction) step introduces variety, resulting in the measure $\left\{\tilde{\mathbf{x}}_{t}^{(i)}, N^{-1}\right\}$, which is an approximation of $p\left(\mathbf{x}_{t} \mid y_{1: t-1}\right)$.

\section{Sequential importance sampling step}

- For $i=1, \ldots, N$, sample from the transition priors

$$
\widetilde{x}_{t}^{(i)} \sim q_{t}\left(\widetilde{x}_{t} \mid x_{0: t-1}^{(i)}, y_{1: t}\right)
$$

and set

$$
\widetilde{x}_{0: t}^{(i)} \triangleq\left(\widetilde{x}_{t}^{(i)}, x_{0: t-1}^{(i)}\right)
$$

- For $i=1, \ldots, N$, evaluate and normalize the importance weights

$$
w_{t}^{(i)} \propto \frac{p\left(y_{t} \mid \widetilde{x}_{t}^{(i)}\right) p\left(\widetilde{x}_{t}^{(i)} \mid x_{0: t-1}^{(i)}, y_{1: t-1}\right)}{q_{t}\left(\widetilde{x}_{t}^{(i)} \mid x_{0: t-1}^{(i)}, y_{1: t}\right)} .
$$

$\underline{\text { Selection step }}$

$$
\begin{aligned}
& \text { - Multiply/Discard particles }\left\{\widetilde{x}_{0: t}^{(i)}\right\}_{i=1}^{N} \text { with high/low importance } \\
& \text { weights } w_{t}^{(i)} \text { to obtain } N \text { particles }\left\{x_{0: t}^{(i)}\right\}_{i=1}^{N} \text {. }
\end{aligned}
$$

Figure 20. Simple SMC algorithm at time $t$. For filtering purposes, there is no need for storing or resampling the past trajectories. 
In generic SMC simulation, one needs to extend the current paths $\left\{x_{0: t-1}^{(i)}\right\}_{i=1}^{N}$ to obtain new paths $\left\{\tilde{x}_{0: t}^{(i)}\right\}_{i=1}^{N}$ using the proposal distribution $q\left(\tilde{x}_{0: t} \mid y_{1: t}\right)$ given by

$$
\left.q\left(\tilde{x}_{0: t} \mid y_{1: t}\right)\right\}=\int q\left(\tilde{x}_{0: t} \mid x_{0: t-1}, y_{1: t}\right) p\left(x_{0: t-1} \mid y_{1: t-1}\right) d x_{0: t-1}
$$

To make this integral tractable, we only propose to modify the particles at time $t$, and leave the past trajectories intact. Consequently

$$
q\left(\tilde{x}_{0: t} \mid y_{1: t}\right)=p\left(x_{0: t-1} \mid y_{1: t-1}\right) q\left(\tilde{x}_{t} \mid x_{0: t-1}, y_{1: t}\right)
$$

The samples from $q(\cdot)$, must be weighted by the importance weights

$$
\begin{aligned}
w_{t} & =\frac{p\left(\tilde{x}_{0: t} \mid y_{1: t}\right)}{q\left(\tilde{x}_{0: t} \mid y_{1: t}\right)}=\frac{p\left(x_{0: t-1} \mid y_{1: t}\right)}{p\left(x_{0: t-1} \mid y_{1: t-1}\right)} \frac{p\left(\tilde{x}_{t} \mid x_{0: t-1}, y_{1: t}\right)}{q\left(\tilde{x}_{t} \mid x_{0: t-1}, y_{1: t}\right)} \\
& \propto \frac{p\left(y_{t} \mid \tilde{x}_{t}\right) p\left(\tilde{x}_{t} \mid x_{0: t-1}, y_{1: t-1}\right)}{q_{t}\left(\tilde{x}_{t} \mid x_{0: t-1}, y_{1: t}\right)}
\end{aligned}
$$

From Eq. (22), we note that the optimal importance distribution is

$$
q\left(\tilde{x}_{t} \mid x_{0: t-1}, y_{1: t}\right)=p\left(\tilde{x}_{t} \mid x_{0: t-1}, y_{1: t}\right) \text {. }
$$

(When using this proposal, one might still encounter difficulties if the ratio of the first two terms of Eq. (22) differs significantly from 1 (Andrieu, Doucet, \& Punskaya, 2001; Pitt \& Shephard, 1999).) The optimal importance distribution can be difficult to evaluate. One can adopt, instead, the transition prior as proposal distribution

$$
q\left(\tilde{x}_{t} \mid x_{0: t-1}, y_{1: t}\right)=p\left(\tilde{x}_{t} \mid x_{0: t-1}, y_{1: t-1}\right)
$$

in which case the importance weights are given by the likelihood function

$$
w_{t} \propto p\left(y_{t} \mid \tilde{x}_{t}\right)
$$

This simplified version of SMC has appeared under many names, including condensation (Isard \& Blake, 1996), survival of the fittest (Kanazawa, Koller, \& Russell, 1995) and the bootstrap filter (Gordon, Salmond, \& Smith, 1993). The importance sampling framework allows us to design more principled and "clever" proposal distributions. For instance, one can adopt suboptimal filters and other approximation methods that make use of the information available at time $t$ to generate the proposal distribution (Doucet, Godsill, \& Andrieu, 2000; de Freitas et al., 2000; Pitt \& Shephard, 1999; van der Merwe et al., 2000). In fact, in some restricted situations, one may interpret the likelihood as a distribution in terms of the states and sample from it directly. In doing so, the importance weights become equal to the transition prior (Fox et al., 2001).

After the importance sampling step, a selection scheme associates to each particle $\tilde{x}_{0: t}^{(i)}$ a number of "children", say $N_{i} \in \mathbb{N}$, such that $\sum_{i=1}^{N} N_{i}=N$. This selection step is what 
allows us to track moving target distributions efficiently by choosing the fittest particles. There are various selection schemes in the literature, but their performance varies in terms of $\operatorname{var}\left[N_{i}\right]$ (Doucet, de Freitas, \& Gordon, 2001).

An important feature of the selection routine is that its interface only depends on particle indices and weights. That is, it can be treated as a black-box routine that does not require any knowledge of what a particle represents (e.g., variables, parameters, models). This enables one to implement variable and model selection schemes straightforwardly. The simplicity of the coding of complex models is, indeed, one of the major advantages of these algorithms.

It is also possible to introduce MCMC steps of invariant distribution $p\left(x_{0: t} \mid y_{1: t}\right)$ on each particle (Andrieu, de Freitas, \& Doucet, 1999; Gilks \& Berzuini, 1998; MacEachern, Clyde, \& Liu, 1999). The basic idea is that if the particles are distributed according to the posterior distribution $p\left(x_{0: t} \mid y_{1: t}\right)$, then applying a Markov chain transition kernel $K\left(x_{0: t}^{\star} \mid x_{0: t}\right)$, with invariant distribution $p\left(\cdot \mid y_{1: t}\right)$ such that $\int K\left(x_{0: t}^{\star} \mid x_{0: t}\right) p\left(x_{0: t} \mid y_{1: t}\right)=p\left(x_{0: t}^{\star} \mid y_{1: t}\right)$, still results in a set of particles distributed according to the posterior of interest. However, the new particles might have been moved to more interesting areas of the state-space. In fact, by applying a Markov transition kernel, the total variation of the current distribution with respect to the invariant distribution can only decrease. Note that we can incorporate any of the standard MCMC methods, such as the Gibbs sampler, MH algorithm and reversible jump MCMC, into the filtering framework, but we no longer require the kernel to be ergodic.

\subsection{The machine learning frontier}

The machine learning frontier is characterised by large dimensional models, massive datasets and many and varied applications. Massive datasets pose no problem in the SMC context. However, in batch MCMC simulation it is often not possible to load the entire dataset into memory. A few solutions based on importance sampling have been proposed recently (Ridgeway, 1999), but there is still great room for innovation in this area.

Despite the auspicious polynomial bounds on the mixing time, it is an arduous task to design efficient samplers in high dimensions. The combination of sampling algorithms with either gradient optimisation or exact methods has proved to be very useful. Gradient optimisation is inherent to Langevin algorithms and hybrid Monte Carlo. These algorithms have been shown to work with large dimensional models such as neural networks (Neal, 1996) and Gaussian processes (Barber \& Williams, 1997). Information about derivatives of the target distribution also forms an integral part of many adaptive schemes, as discussed in Section 2.3. Recently, it has been argued that the combination of MCMC and variational optimisation techniques can also lead to more efficient sampling (de Freitas et al., 2001).

The combination of exact inference with sampling methods within the framework of RaoBlackwellisation (Casella \& Robert, 1996) can also result in great improvements. Suppose we can divide the hidden variables $x$ into two groups, $u$ and $v$, such that $p(x)=p(v \mid u) p(u)$ and, conditional on $u$, the conditional posterior distribution $p(v \mid u)$ is analytically tractable. Then we can easily marginalise out $v$ from the posterior, and only need to focus on sampling from $p(u)$, which lies in a space of reduced dimension. That is, we sample $u^{(i)} \sim p(u)$ and 
then use exact inference to compute

$$
p(v)=\frac{1}{N} \sum_{i=1}^{N} p\left(v \mid u^{(i)}\right)
$$

By identifying "troublesome" variables and sampling them, the rest of the problem can often be solved easily using exact algorithms such as Kalman filters, HMMs or junction trees. For example, one can apply this technique to sample variables that eliminate loops in graphical models and then compute the remaining variables with efficient analytical algorithms (Jensen, Kong, \& Kjærulff, 1995; Wilkinson \& Yeung, 2002). Other application areas include dynamic Bayesian networks (Doucet et al., 2000), conditionally Gaussian models (Carter \& Kohn, 1994; De Jong \& Shephard, 1995; Doucet, 1998) and model averaging for graphical models (Friedman \& Koller, this issue). The problem of how to automatically identify which variables should be sampled, and which can be handled analytically is still open. An interesting development is the augmentation of high dimensional models with low dimensional artificial variables. By sampling only the artificial variables, the original model decouples into simpler, more tractable submodels (Albert \& Chib, 1993; Andrieu, de Freitas, \& Doucet, 2001b; Wood \& Kohn, 1998); see also Holmes and Denison (this issue). This strategy allows one to map probabilistic classification problems to simpler regression problems.

The design of efficient sampling methods most of the times hinges on awareness of the basic building blocks of MCMC (mixtures of kernels, augmentation strategies and blocking) and on careful design of the proposal mechanisms. The latter requires domain specific knowledge and heuristics. There are great opportunities for combining existing sub-optimal algorithms with MCMC in many machine learning problems. Some areas that are already benefiting from sampling methods include:

1. Computer vision. Tracking (Isard \& Blake, 1996; Ormoneit, Lemieux, \& Fleet, 2001), stereo matching (Dellaert et al., this issue), colour constancy (Forsyth, 1999), restoration of old movies (Morris, Fitzgerald, \& Kokaram, 1996) and segmentation (Clark \& Quinn, 1999; Kam, 2000; Tu \& Zhu, 2001).

2. Web statistics. Estimating coverage of search engines, proportions belonging to specific domains and the average size of web pages (Bar-Yossef et al., 2000).

3. Speech and audio processing. Signal enhancement (Godsill \& Rayner, 1998; Vermaak et al., 1999).

4. Probabilistic graphical models. For example (Gilks, Thomas, \& Spiegelhalter, 1994; Wilkinson \& Yeung, 2002) and several papers in this issue.

5. Regression and classification. Neural networks and kernel machines (Andrieu, de Freitas, \& Doucet, 2001a; Holmes \& Mallick, 1998; Neal, 1996; Müller \& Rios Insua, 1998), Gaussian processes (Barber \& Williams, 1997), CART (Denison, Mallick, \& Smith, 1998) and MARS (Holmes \& Denison, this issue).

6. Computer graphics. Light transport (Veach \& Guibas, 1997) and sampling plausible solutions to multi-body constraint problems (Chenney \& Forsyth, 2000). 
7. Data association. Vehicle matching in highway systems (Pasula et al., 1999) and multitarget tracking (Bergman, 1999).

8. Decision theory. Partially observable Markov decision Processes (POMDPs) (Thrun, 2000; Salmond \& Gordon, 2001), abstract Markov policies (Bui, Venkatesh, \& West, 1999) and influence diagrams (Bielza, Müller, \& Rios Insua, 1999).

9. First order probabilistic logic. (Pasula \& Russell, 2001).

10. Genetics and molecular biology. DNA microarray data (West et al., 2001), cancer gene mapping (Newton \& Lee, 2000), protein alignment (Neuwald et al., 1997) and linkage analysis (Jensen, Kong, \& Kjærulff, 1995).

11. Robotics. Robot localisation and map building (Fox et al., 2001).

12. Classical mixture models. Mixtures of independent factor analysers (Utsugi, 2001) and mixtures of factor analysers (Fokoué \& Titterington, this issue).

We hope that this review will be a useful resource to people wishing to carry out further research at the interface between MCMC and machine learning. For conciseness, we have skipped many interesting ideas, including tempering and coupling. For more details, we advise the readers to consult the references at the end of this paper.

\section{Acknowledgments}

We would like to thank Robin Morris, Kevin Murphy, Mark Paskin, Sekhar Tatikonda and Mike Titterington.

\section{References}

Al-Qaq, W. A., Devetsikiotis, M., \& Townsend, J. K. (1995). Stochastic gradient optimization of importance sampling for the efficient simulation of digital communication systems. IEEE Transactions on Communications, 43:12, 2975-2985.

Albert, J., \& Chib, S. (1993). Bayesian analysis of binary and polychotomous response data. Journal of the American Statistical Association, 88:422, 669-679.

Anderson, H. L. (1986). Metropolis, Monte Carlo, and the MANIAC. Los Alamos Science, 14, 96-108.

Andrieu, C., \& Doucet, A. (1999). Joint Bayesian detection and estimation of noisy sinusoids via reversible jump MCMC. IEEE Transactions on Signal Processing, 47:10, 2667-2676.

Andrieu, C., Breyer, L. A., \& Doucet, A. (1999). Convergence of simulated annealing using Foster-Lyapunov criteria. Technical Report CUED/F-INFENG/TR 346, Cambridge University Engineering Department.

Andrieu, C., de Freitas, N., \& Doucet, A. (1999). Sequential MCMC for Bayesian model selection. In IEEE Higher Order Statistics Workshop, Caesarea, Israel (pp. 130-134).

Andrieu, C., de Freitas, N., \& Doucet, A. (2000a). Reversible jump MCMC simulated annealing for neural networks. In Uncertainty in artificial intelligence (pp. 11-18). San Mateo, CA: Morgan Kaufmann.

Andrieu, C., de Freitas, N., \& Doucet, A. (2000b). Robust full Bayesian methods for neural networks. In S. A. Solla, T. K. Leen, \& K.-R. Müller (Eds.), Advances in neural information processing systems 12 (pp. 379-385). MIT Press.

Andrieu, C., de Freitas, N., \& Doucet, A. (2001a). Robust full Bayesian learning for radial basis networks. Neural Computation, 13:10, 2359-2407.

Andrieu, C., de Freitas, N., \& Doucet, A. (2001b). Rao-blackwellised particle filtering via data augmentation. Advances in Neural Information Processing Systems (NIPS13). 
Andrieu, C., Doucet, A., \& Punskaya, E. (2001). Sequential Monte Carlo methods for optimal filtering. In A Doucet, N. de Freitas, \& N. J. Gordon (Eds.), Sequential Monte Carlo methods in practice. Berlin: SpringerVerlag.

Applegate, D., \& Kannan, R. (1991). Sampling and integration of near log-concave functions. In Proceedings of the Twenty Third Annual ACM Symposium on Theory of Computing (pp. 156-163).

Bar-Yossef, Z., Berg, A., Chien, S., Fakcharoenphol, J., \& Weitz, D. (2000). Approximating aggregate queries about web pages via random walks. In International Conference on Very Large Databases (pp. 535-544).

Barber, D., \& Williams, C. K. I. (1997). Gaussian processes for Bayesian classification via hybrid Monte Carlo. In M. C. Mozer, M. I. Jordan, \& T. Petsche (Eds.), Advances in neural information processing systems 9 (pp. 340-346). Cambridge, MA: MIT Press.

Baum, L. E., Petrie, T., Soules, G., \& Weiss, N. (1970). A maximization technique occurring in the statistical analysis of probabilistic functions of Markov chains. Annals of Mathematical Statistics, 41, 164-171.

Baxter, R. J. (1982). Exactly solved models in statistical mechanics. San Diego, CA: Academic Press.

Beichl, I., \& Sullivan, F. (2000). The Metropolis algorithm. Computing in Science \& Engineering, 2:1, 65-69.

Bergman, N. (1999). Recursive Bayesian estimation: Navigation and tracking applications. Ph.D. Thesis, Department of Electrical Engineering, Linköping University, Sweden.

Berners-Lee, T., Cailliau, R., Luotonen, A., Nielsen, H. F., \& Secret, A. (1994). The World-Wide Web. Communications of the ACM, 10:4, 49-63.

Besag, J., Green, P. J., Hidgon, D., \& Mengersen, K. (1995). Bayesian computation and stochastic systems. Statistical Science, 10:1, 3-66.

Bielza, C., Müller, P., \& Rios Insua, D. (1999). Decision Analysis by Augmented Probability Simulation, Management Science, 45:7, 995-1007.

Brooks, S. P. (1998). Markov chain Monte Carlo method and its application. The Statistician, 47:1, 69-100.

Browne, W. J., \& Draper, D. (2000). Implementation and performance issues in the Bayesian and likelihood fitting of multilevel models. Computational Statistics, 15, 391-420.

Bucher, C. G. (1988). Adaptive sampling-An iterative fast Monte Carlo procedure. Structural Safety, 5, $119-126$.

Bui, H. H., Venkatesh, S., \& West, G. (1999). On the recognition of abstract Markov policies. In National Conference on Artificial Intelligence (AAAI-2000).

Carlin, B. P., \& Chib, S. (1995). Bayesian Model choice via MCMC. Journal of the Royal Statistical Society Series $B, 57,473-484$.

Carter, C. K., \& Kohn, R. (1994). On Gibbs sampling for state space models. Biometrika, 81:3, 541-553.

Casella, G., \& Robert, C. P. (1996). Rao-Blackwellisation of sampling schemes. Biometrika, 83:1, 81-94.

Casella, G., Mengersen, K. L., Robert, C. P., \& Titterington, D. M. (1999). Perfect slice samplers for mixtures of distributions. Technical Report BU-1453-M, Department of Biometrics, Cornell University.

Celeux, G., \& Diebolt, J. (1985). The SEM algorithm: A probabilistic teacher algorithm derived from the EM algorithm for the mixture problem. Computational Statistics Quarterly, 2, 73-82.

Celeux, G., \& Diebolt, J. (1992). A stochastic approximation type EM algorithm for the mixture problem. Stochastics and Stochastics Reports, 41, 127-146.

Chen, M. H., Shao, Q. M., \& Ibrahim, J. G. (Eds.) (2001). Monte Carlo methods for Bayesian computation. Berlin: Springer-Verlag.

Cheng, J., \& Druzdzel, M. J. (2000). AIS-BN: An adaptive importance sampling algorithm for evidential reasoning in large bayesian networks. Journal of Artificial Intelligence Research, 13, 155-188.

Chenney, S., \& Forsyth, D. A. (2000). Sampling plausible solutions to multi-body constraint problems. SIGGRAPH (pp. 219-228).

Clark, E., \& Quinn, A. (1999). A data-driven Bayesian sampling scheme for unsupervised image segmentation. In IEEE International Conference on Acoustics, Speech, and Signal Processing, Arizona (Vol. 6, pp. 34973500).

Damien, P., Wakefield, J., \& Walker, S. (1999). Gibbs sampling for Bayesian non-conjugate and hierarchical models by auxiliary variables. Journal of the Royal Statistical Society B, 61:2, 331-344.

de Freitas, N., Højen-Sørensen, P., Jordan, M. I., \& Russell, S. (2001). Variational MCMC. In J. Breese \& D. Koller (Eds.), Uncertainty in artificial intelligence (pp. 120-127). San Matio, CA: Morgan Kaufmann.

de Freitas, N., Niranjan, M., Gee, A. H., \& Doucet, A. (2000). Sequential Monte Carlo methods to train neural network models. Neural Computation, 12:4, 955-993. 
De Jong, P., \& Shephard, N. (1995). Efficient sampling from the smoothing density in time series models. Biometrika, 82:2, 339-350.

Dempster, A. P., Laird, N. M., \& Rubin, D. B. (1997). Maximum likelihood from incomplete data via the EM algorithm. Journal of the Royal Statistical Society Series B, 39, 1-38.

Denison, D. G. T., Mallick, B. K., \& Smith, A. F. M. (1998). A Bayesian CART algorithm. Biometrika, 85, 363-377.

Diaconis, P., \& Saloff-Coste, L. (1998). What do we know about the Metropolis algorithm? Journal of Computer and System Sciences, 57, 20-36.

Doucet, A. (1998). On sequential simulation-based methods for Bayesian filtering. Technical Report CUED/FINFENG/TR 310, Department of Engineering, Cambridge University.

Doucet, A., de Freitas, N., \& Gordon, N. J. (Eds.) (2001). Sequential Monte Carlo methods in practice. Berlin: Springer-Verlag.

Doucet, A., de Freitas, N., Murphy, K., \& Russell, S. (2000). Rao blackwellised particle filtering for dynamic Bayesian networks. In C. Boutilier \& M. Godszmidt (Eds.), Uncertainty in artificial intelligence (pp. 176-183). Morgan Kaufmann Publishers.

Doucet, A., Godsill, S., \& Andrieu, C. (2000). On sequential Monte Carlo sampling methods for Bayesian filtering. Statistics and Computing, 10:3, 197-208.

Doucet, A., Godsill, S. J., \& Robert, C. P. (2000). Marginal maximum a posteriori estimation using MCMC. Technical Report CUED/F-INFENG/TR 375, Cambridge University Engineering Department.

Duane, S., Kennedy, A. D., Pendleton, B. J., \& Roweth, D. (1987). Hybrid Monte Carlo. Physics Letters B, 195:2, 216-222.

Dyer, M., Frieze, A., \& Kannan, R. (1991). A random polynomial-time algorithm for approximating the volume of convex bodies. Journal of the ACM, 1:38, 1-17.

Eckhard, R. (1987). Stan Ulam, John Von Neumann and the Monte Carlo method. Los Alamos Science, 15, 131-136.

Escobar, M. D., \& West, M. (1995). Bayesian density estimation and inference using mixtures. Journal of the American Statistical Association, 90, 577-588.

Fill, J. A. (1998). An interruptible algorithm for perfect sampling via Markov chains. The Annals of Applied Probability, 8:1, 131-162.

Forsyth, D. A. (1999). Sampling, resampling and colour constancy. In IEEE Conference on Computer Vision and Pattern Recognition (pp. 300-305).

Fox, D., Thrun, S., Burgard, W., \& Dellaert, F. (2001). Particle filters for mobile robot localization. In A. Doucet, N. de Freitas, \& N. J. Gordon (Eds.), Sequential Monte Carlo methods in practice. Berlin: Springer-Verlag.

Gelfand, A. E., \& Sahu, S. K. (1994). On Markov chain Monte Carlo acceleration. Journal of Computational and Graphical Statistics, 3, 261-276.

Gelfand, A. E., \& Smith, A. F. M. (1990). Sampling-based approaches to calculating marginal densities. Journal of the American Statistical Association, 85:410, 398-409.

Geman, S., \& Geman, D. (1984). Stochastic relaxation, Gibbs distributions and the Bayesian restoration of images. IEEE Transactions on Pattern Analysis and Machine Intelligence, 6:6, 721-741.

Geweke, J. (1989). Bayesian inference in econometric models using Monte Carlo integration. Econometrica, 24, 1317-1399.

Ghahramani, Z. (1995). Factorial learning and the EM algorithm. In G. Tesauro, D. S. Touretzky, \& J. Alspector (Eds.), Advances in neural information processing systems 7 (pp. 617-624).

Ghahramani, Z., \& Jordan, M. (1995). Factorial hidden Markov models. Technical Report 9502, MIT Artificial Intelligence Lab, MA.

Gilks, W. R., \& Berzuini, C. (1998). Monte Carlo inference for dynamic Bayesian models. Unpublished. Medical Research Council, Cambridge, UK.

Gilks, W. R., \& Roberts, G. O. (1996). Strategies for improving MCMC. In W. R. Gilks, S. Richardson, \& D. J. Spiegelhalter (Eds.), Markov chain Monte Carlo in practice (pp. 89-114). Chapman \& Hall.

Gilks, W. R., Richardson, S., \& Spiegelhalter, D. J. (Eds.) (1996). Markov chain Monte Carlo in practice. Suffolk: Chapman and Hall.

Gilks, W. R., Roberts, G. O., \& Sahu, S. K. (1998). Adaptive Markov chain Monte Carlo through regeneration. Journal of the American Statistical Association, 93, 763-769. 
Gilks, W. R., Thomas, A., \& Spiegelhalter, D. J. (1994). A language and program for complex Bayesian modelling. The Statistician, 43, 169-178.

Godsill, S. J., \& Rayner, P. J. W. (Eds.) (1998). Digital audio restoration: A statistical model based approach. Berlin: Springer-Verlag.

Gordon, N. J., Salmond, D. J., \& Smith, A. F. M. (1993). Novel approach to nonlinear/non-Gaussian Bayesian state estimation. IEE Proceedings-F, 140:2, 107-113.

Green, P. J. (1995). Reversible jump Markov chain Monte Carlo computation and Bayesian model determination. Biometrika, 82, 711-732.

Green, P. J., \& Richardson, S. (2000). Modelling heterogeneity with and without the Dirichlet process. Department of Statistics, Bristol University.

Haario, H., \& Sacksman, E. (1991). Simulated annealing process in general state space. Advances in Applied Probability, 23, 866-893.

Hastings, W. K. (1970). Monte Carlo sampling methods using Markov chains and their Applications. Biometrika 57, 97-109.

Higdon, D. M. (1998). Auxiliary variable methods for Markov chain Monte Carlo with application. Journal of American Statistical Association, 93:442, 585-595.

Holmes, C. C., \& Mallick, B. K. (1998). Bayesian radial basis functions of variable dimension. Neural Computation, $10: 5,1217-1233$.

Isard, M., \& Blake, A. (1996). Contour tracking by stochastic propagation of conditional density. In European Conference on Computer Vision (pp. 343-356). Cambridge, UK.

Ishwaran, H. (1999). Application of hybrid Monte Carlo to Bayesian generalized linear models: Quasicomplete separation and neural networks. Journal of Computational and Graphical Statistics, 8, 779-799.

Jensen, C. S., Kong, A., \& Kjærulff, U. (1995). Blocking-Gibbs sampling in very large probabilistic expert systems. International Journal of Human-Computer Studies, 42, 647-666.

Jerrum, M., \& Sinclair, A. (1996). The Markov chain Monte Carlo method: an approach to approximate counting and integration. In D. S. Hochbaum (Ed.), Approximation algorithms for NP-hard problems (pp. 482-519). PWS Publishing.

Jerrum, M., Sinclair, A., \& Vigoda, E. (2000). A polynomial-time approximation algorithm for the permanent of a matrix. Technical Report TR00-079, Electronic Colloquium on Computational Complexity.

Kalos, M. H., \& Whitlock, P. A. (1986). Monte Carlo methods. New York: John Wiley \& Sons.

Kam, A. H. (2000). A general multiscale scheme for unsupervised image segmentation. Ph.D. Thesis, Department of Engineering, Cambridge University, Cambridge, UK.

Kanazawa, K., Koller, D., \& Russell, S. (1995). Stochastic simulation algorithms for dynamic probabilistic networks. In Proceedings of the Eleventh Conference on Uncertainty in Artificial Intelligence (pp. 346-351). Morgan Kaufmann.

Kannan, R., \& Li, G. (1996). Sampling according to the multivariate normal density. In 37th Annual Symposium on Foundations of Computer Science (pp. 204-212). IEEE.

Kirkpatrick, S., Gelatt, C. D., \& Vecchi, M. P. (1983). Optimization by simulated annealing. Science, 220, 671680.

Levine, R., \& Casella, G. (2001). Implementations of the Monte Carlo EM algorithm. Journal of Computational and Graphical Statistics, 10:3, 422-440.

Liu, J. S. (Ed.) (2001). Monte Carlo strategies in scientific computing. Berlin: Springer-Verlag.

MacEachern, S. N., Clyde, M., \& Liu, J. S. (1999). Sequential importance sampling for nonparametric Bayes models: The next generation. Canadian Journal of Statistics, 27, 251-267.

McCulloch, C. E. (1994). Maximum likelihood variance components estimation for binary data. Journal of the American Statistical Association, 89:425, 330-335.

Mengersen, K. L., \& Tweedie, R. L. (1996). Rates of convergence of the Hastings and Metropolis algorithms. The Annals of Statistics, 24, 101-121.

Metropolis, N., \& Ulam, S. (1949). The Monte Carlo method. Journal of the American Statistical Association, 44:247, 335-341.

Metropolis, N., Rosenbluth, A. W., Rosenbluth, M. N., Teller, A. H., \& Teller, E. (1953). Equations of state calculations by fast computing machines. Journal of Chemical Physics, 21, 1087-1091.

Meyn, S. P., \& Tweedie, R. L. (1993). Markov chains and stochastic stability. New York: Springer-Verlag. 
Mira, A. (1999). Ordering, slicing and splitting Monte Carlo Markov chains. Ph.D. Thesis, School of Statistics, University of Minnesota.

Morris, R. D., Fitzgerald, W. J., \& Kokaram, A. C. (1996). A sampling based approach to line scratch removal from motion picture frames. In IEEE International Conference on Image Processing (pp. 801-804).

Müller, P., \& Rios Insua, D. (1998). Issues in Bayesian analysis of neural network models. Neural Computation, 10, 571-592.

Mykland, P., Tierney, L., \& Yu, B. (1995). Regeneration in Markov chain samplers. Journal of the American Statistical Association, 90, 233-241.

Neal, R. M. (1993). Probabilistic inference using markov chain monte carlo methods. Technical Report CRG-TR93-1, Dept. of Computer Science, University of Toronto.

Neal, R. M. (1996). Bayesian learning for neural networks. Lecture Notes in Statistics No. 118. New York: Springer-Verlag.

Neal, R. M. (2000). Slice sampling. Technical Report No. 2005, Department of Statistics, University of Toronto.

Neuwald, A. F., Liu, J. S., Lipman, D. J., \& Lawrence, C. E. (1997). Extracting protein alignment models from the sequence database. Nucleic Acids Research, 25:9, 1665-1677.

Newton, M. A., \& Lee, Y. (2000). Inferring the location and effect of tumor suppressor genes by instability-selection modeling of allelic-loss data. Biometrics, 56, 1088-1097.

Ormoneit, D., Lemieux, C., \& Fleet, D. (2001). Lattice particle filters. Uncertainty in artificial intelligence. San Mateo, CA: Morgan Kaufmann.

Ortiz, L. E., \& Kaelbling, L. P. (2000). Adaptive importance sampling for estimation in structured domains. In C. Boutilier, \& M. Godszmidt (Eds.), Uncertainty in artificial intelligence (pp. 446-454). San Mateo, CA: Morgan Kaufmann Publishers.

Page, L., Brin, S., Motwani, R., \& Winograd, T. (1998). The PageRank citation ranking: Bringing order to the Web. Stanford Digital Libraries Working Paper.

Pasula, H., \& Russell, S. (2001). Approximate inference for first-order probabilistic languages. In International Joint Conference on Artificial Intelligence, Seattle.

Pasula, H., Russell, S., Ostland, M., \& Ritov, Y. (1999). Tracking many objects with many sensors. In International Joint Conference on Artificial Intelligence, Stockholm.

Pearl, J. (1987). Evidential reasoning using stochastic simulation. Artificial Intelligence, 32, 245-257.

Peskun, P. H. (1973). Optimum Monte-Carlo sampling using Markov chains. Biometrika, 60:3, 607-612.

Pitt, M. K., \& Shephard, N. (1999). Filtering via simulation: Auxiliary particle filters. Journal of the American Statistical Association, 94:446, 590-599.

Propp, J., \& Wilson, D. (1998). Coupling from the past: a user's guide. In D. Aldous, \& J. Propp (Eds.), Microsurveys in discrete probability. DIMACS series in discrete mathematics and theoretical computer science.

Remondo, D., Srinivasan, R., Nicola, V. F., van Etten, W. C., \& Tattje, H. E. P. (2000). Adaptive importance sampling for performance evaluation and parameter optimization of communications systems. IEEE Transactions on Communications, 48:4, 557-565.

Richardson, S., \& Green, P. J. (1997). On Bayesian analysis of mixtures with an unknown number of components. Journal of the Royal Statistical Society B, 59:4, 731-792.

Ridgeway, G. (1999). Generalization of boosting algorithms and applications of bayesian inference for massive datasets. Ph.D. Thesis, Department of Statistics, University of Washington.

Rios Insua, D., \& Müller, P. (1998). Feedforward neural networks for nonparametric regression. In D. K. Dey, P. Müller, \& D. Sinha (Eds.), Practical nonparametric and semiparametric bayesian statistics (pp. 181-191). Springer Verlag.

Robert, C. P., \& Casella, G. (1999). Monte Carlo statistical methods. New York: Springer-Verlag.

Roberts, G., \& Tweedie, R. (1996). Geometric convergence and central limit theorems for multidimensional Hastings and Metropolis algorithms. Biometrika, 83, 95-110.

Rubin, D. B. (1998). Using the SIR algorithm to simulate posterior distributions. In J. M. Bernardo, M. H. DeGroot, D. V. Lindley, \& A. F. M. Smith (Eds.), Bayesian statistics 3 (pp. 395-402). Cambridge, MA: Oxford University Press.

Rubinstein, R. Y. (Eds.) (1981). Simulation and the Monte Carlo method. New York: John Wiley and Sons.

Salmond, D., \& Gordon, N. (2001). Particles and mixtures for tracking and guidance. In A. Doucet, N. de Freitas, \& N. J. Gordon (Eds.), Sequential Monte Carlo methods in practice. Berlin: Springer-Verlag. 
Schuurmans, D., \& Southey, F. (2000). Monte Carlo inference via greedy importance sampling. In C. Boutilier, \& M. Godszmidt (Eds.), Uncertainty in artificial intelligence (pp. 523-532). Morgan Kaufmann Publishers. Sherman, R. P., Ho, Y. K., \& Dalal, S. R. (1999). Conditions for convergence of Monte Carlo EM sequences with an application to product diffusion modeling. Econometrics Journal, 2:2, 248-267.

Smith, P. J., Shafi, M., \& Gao, H. (1997). Quick simulation: A review of importance sampling techniques in communications systems. IEEE Journal on Selected Areas in Communications, 15:4, 597-613.

Stephens, M. (1997). Bayesian methods for mixtures of normal distributions. Ph.D. Thesis, Department of Statistics, Oxford University, England.

Swendsen, R. H., \& Wang, J. S. (1987). Nonuniversal critical dynamics in Monte Carlo simulations. Physical Review Letters, 58:2, 86-88.

Tanner, M. A., \& Wong, W. H. (1987). The calculation of posterior distributions by data augmentation. Journal of the American Statistical Association, 82:398, 528-550.

Thrun, S. (2000). Monte Carlo POMDPs. In S. Solla, T. Leen, \& K.-R. Müller (Eds.), Advances in neural information processing systems 12 (pp. 1064-1070). Cambridge, MA: MIT Press.

Tierney, L. (1994). Markov chains for exploring posterior distributions. The Annals of Statistics, 22:4, 1701-1762.

Tierney, L., \& Mira, A. (1999). Some adaptive Monte Carlo methods for Bayesian inference. Statistics in Medicine, $18,2507-2515$.

Troughton, P. T., \& Godsill, S. J. (1998). A reversible jump sampler for autoregressive time series. In International Conference on Acoustics, Speech and Signal Processing (Vol. IV, pp. 2257-2260).

Tu, Z. W., \& Zhu, S. C. (2001). Image segmentation by data driven Markov chain Monte Carlo. In International Computer Vision Conference.

Utsugi, A. (2001). Ensemble of independent factor analyzers with application to natural image analysis. Neural Processing Letters, 14:1, 49-60.

van der Merwe, R., Doucet, A., de Freitas, N., \& Wan, E. (2000). The unscented particle filter. Technical Report CUED/F-INFENG/TR 380, Cambridge University Engineering Department.

Van Laarhoven, P. J., \& Arts, E. H. L. (1987). Simulated annealing: Theory and applications. Amsterdam: Reidel Publishers.

Veach, E., \& Guibas, L. J. (1997). Metropolis light transport. SIGGRAPH, 31, 65-76.

Vermaak, J., Andrieu, C., Doucet, A., \& Godsill, S. J. (1999). Non-stationary Bayesian modelling and enhancement of speech signals. Technical Report CUED/F-INFENG/TR, Cambridge University Engineering Department.

Wakefield, J. C., Gelfand, A. E., \& Smith, A. F. M. (1991). Efficient generation of random variates via the ratio-of-uniforms methods. Statistics and Computing, 1, 129-133.

Wei, G. C. G., \& Tanner, M. A. (1990). A Monte Carlo implementation of the EM algorithm and the poor man's data augmentation algorithms. Journal of the American Statistical Association, 85:411, 699-704.

West, M., Nevins, J. R., Marks, J. R., Spang, R., \& Zuzan, H. (2001). Bayesian regression analysis in the "large p, small n" paradigm with application in DNA microarray studies. Department of Statistics, Duke University.

Wilkinson, D. J., \& Yeung, S. K. H. (2002). Conditional simulation from highly structured Gaussian systems, with application to blocking-MCMC for the Bayesian analysis of very large linear models. Statistics and Computing, 12, 287-300

Wood, S., \& Kohn, R. (1998). A Bayesian approach to robust binary nonparametric regression. Journal of the American Statistical Association, 93:441, 203-213. 